\title{
Crack initiation and propagation in unreinforced masonry specimens subjected to repeated in-plane loading during light damage
}

\author{
Paul A. Korswagen ${ }^{1}$ (D) $\cdot$ Michele Longo ${ }^{1} \cdot$ Edwin Meulman $^{1} \cdot$ Jan G. Rots $^{1}$
}

Received: 27 September 2018 / Accepted: 27 December 2018 / Published online: 2 January 2019

(c) The Author(s) 2019, corrected publication 2019

\begin{abstract}
In recent years, gas extraction in the northern part of the Netherlands has been causing low-magnitude, induced, shallow earthquakes. Besides safety, the prediction and evaluation of 'light' damage due to these induced ground motions is important, as it is related to economic and serviceability losses, and societal unrest. An experimental and numerical campaign is ongoing at Delft University of Technology, aiming to improve the knowledge of the underlying physics of crack initiation and propagation in unreinforced masonry (URM) structures typical in the Netherlands. A damage scale and damage parameter are defined herein in order to objectively quantify cracking damage as a function of the number, length, and width of cracks in masonry walls. The cracking mechanisms are studied for URM walls and spandrels subjected to in-plane loading. Displacements, strains, and loads under which cracking starts and propagates are evaluated and correlations are sought. The Digital Image Correlation measuring system is used to accurately detect crack formation and the evolution of the cracking pattern. This is also utilised to validate and calibrate nonlinear finite element models. From the experiments, drift values are obtained for the light damage state of the masonry walls. A range between $0.3 \%$ and $1.1 \%$ is set as belonging to light damage. Moreover, a damage accumulation or material degradation was observed during cyclic testing. Additionally, fracture-mechanics based, micro and macro finite element models are capable of reproducing the repetitive behaviour of the tests.
\end{abstract}

Keywords Unreinforced masonry structures · Earthquake damage $\cdot$ Crack analysis · Digital Image Correlation · Damage scale $\cdot$ Material degradation

\section{Introduction}

The extraction of natural gas from the region of Groningen, The Netherlands, in the past decades has given rise to human-induced seismic activity (NAM 2013). These earthquakes of moderate magnitude have up until now lead to relatively low vibration levels

Paul A. Korswagen

p.a.korswageneguren@tudelft.nl

1 Delft University of Technology, Stevinweg 1, 2628 CN Delft, The Netherlands 
with registered values of ground accelerations up to a maximum of $0.11 \%$ of gravity (as recorded in 2018 during the 'Zeerijp' earthquake). Higher horizontal ground acceleration values are possible (Spetzler and Dost 2017) and these pose a risk to the existing building stock, most of which are unprepared for earthquakes (NAM 2016). Consequently, multiple studies have focused on assessing the ultimate, near-collapse capacity (e.g. Esposito et al. 2016, 2018; Messali and Rots 2018; Tomassetti et al. 2017; Graziotti et al. 2017) and the associated risk (e.g. Noorlandt et al. 2018; Bommer et al. 2017) of the predominant, unreinforced masonry of the region.

However, while the larger seismic events may carry a greater risk, the more frequent, light earthquakes are believed to be linked to minor, aesthetic damage (denoted in this context as damage state one-DS1, see for instance de Vent et al. 2011) present in a number of masonry structures across the Groningen region (see for instance, Van Staalduinen et al. 2018). This 'light damage' has been observed to be related to economic and serviceability losses, and societal unrest. Recent efforts have started to focus, albeit with an empirical approach, also on these minor damage states potentially caused by the lighter, more frequent events (see for example, Crowley et al. 2018); however, the influence of these more frequent earthquakes, leading to low ground vibration values, has yet to be studied as extensively, in particular from a physical perspective.

Tectonic and induced earthquakes do not differ significantly in terms of duration, frequency and other seismic characterisation parameters affecting structural performance (Bal et al. 2018). Yet, building practices in areas where seismic events have always been of concern, differ considerably from those where earthquakes have become new phenomena. This significantly reduces the literature applicable to the analysis of these buildings.

Typical masonry in the area of Groningen is unreinforced, slender, and often shows poor bonding between mortar and bricks (Arup 2013; Jafari and Esposito 2019). This makes their behaviour similar to monumental masonry buildings as found in Italy (e.g. Castellazzi et al. 2017) or Canada (e.g. Elmenshawi et al. 2010; Abo-El-Ezz et al. 2013), or to masonry as (non-)structural in-fills (Colangelo 2015). Nevertheless, insight into the minor damage state (DS1) is scarce.

The region of Basel, in Switzerland, was also affected by induced seismicity, in this case, due to geothermal energy production (Dyer et al. 2008). The larger events lead to peak ground velocity values of around $5 \mathrm{~mm} / \mathrm{s}$ (which can be viewed as low vibration values); hence, many similarities can be drawn to the situation in Groningen when considering the lighter seismic events. Recent efforts to better understand the seismicity on Basel (Mignan et al. 2015) also incorporate the study of minor damage to masonry structures (Didier et al. 2017). Didier et al. (2018) observe 'damage' experimentally in plastered masonry panels using Digital Image Correlation (DIC). Nonetheless, their tests show cracks cutting through the masonry bricks, which has seldom been observed in the Groningen region (Van Staalduinen et al. 2018) and is not representative of the poor-bond masonry where cracks mainly localise at the interface between mortar and brick (e.g. Jafari et al. 2017).

In addition, the soil in Groningen is of the soft type and varies locally with different layers of clay, peat, and sand (see e.g. Kruiver et al. 2017). This raises the interaction of damage due to differential settlements and cracking due to seismicity as an important consideration. Furthermore, in Groningen, light damage appears to be limited to in-plane effects with no clear evidence of damage due to out-of-plane effects (Van Staalduinen et al. 2018).

Consequently, to observe the influence of frequent, light earthquakes on masonry structures in Groningen, in the Netherlands, experimental tests have been conducted at Delft University of Technology. The goal is to investigate the phenomena of crack initiation and propagation in masonry for recent, induced earthquakes with an in-depth look into crack 
patterns, crack widths, and the effect of repetitive and combined loadings. The tests are used to calibrate finite element models, capable of further analysing the progression of damage in more complex situations such as the combined actions of seismicity and differential settlements or differential thermal or hygral movements. Furthermore, just as Messali and Rots (2018) sets drift limits for the ultimate limit state capacity of masonry walls, this paper seeks to offer a first insight into drift values that correspond to the serviceability limit state (light damage).

In this article, the case of an in-plane loaded, sample masonry wall subjected to an overburden is studied (see Fig. 1). This case was selected to study horizontal and diagonal cracks. The single-wythe wall of $100 \mathrm{~mm}$ in thickness was built with clay bricks and cement mortar, replicating masonry properties of the period before 1950 in the Netherlands (see Jafari et al. 2017; Esposito et al. 2016). An overview of these properties is provided in Table 7 in the Appendix to this paper. Additionally, vertical cracks in spandrels of the same material are studied via modified four-point-bending tests.

The outline of this paper is as follows.

When assessing light damage, a quantitative definition, tailored to evaluating the intensity and the progression of damage on these walls, is paramount. Such a damage parameter is herein formulated and is presented at the start of this paper in Sect. 2.

Then, the experimental setups for the tests treated in this article are described in Sect. 3. First, the setup for in-plane loading of the full-scale walls (Fig. 1) is presented; while a modified four-point-bending setup, used for assaying vertical cracks in spandrels, is described later. Additionally, the specifics of the DIC measuring technique are described; and, the reasoning for choosing a repetitive and a cyclic loading history is treated.

In Sect. 4, the results of the aforementioned tests are presented. The first part is devoted to the results of the three specimens of the selected full-scale wall geometry, tested under controlled, one- and two-way cyclic top lateral displacement deformation, whereas the second part is dedicated to the modified four-point-bending tests on spandrels.

Section 5 concerns the calibration of the finite element models against the laboratory experiments. These models were calibrated in terms of stiffness, strength, crack patterns, and

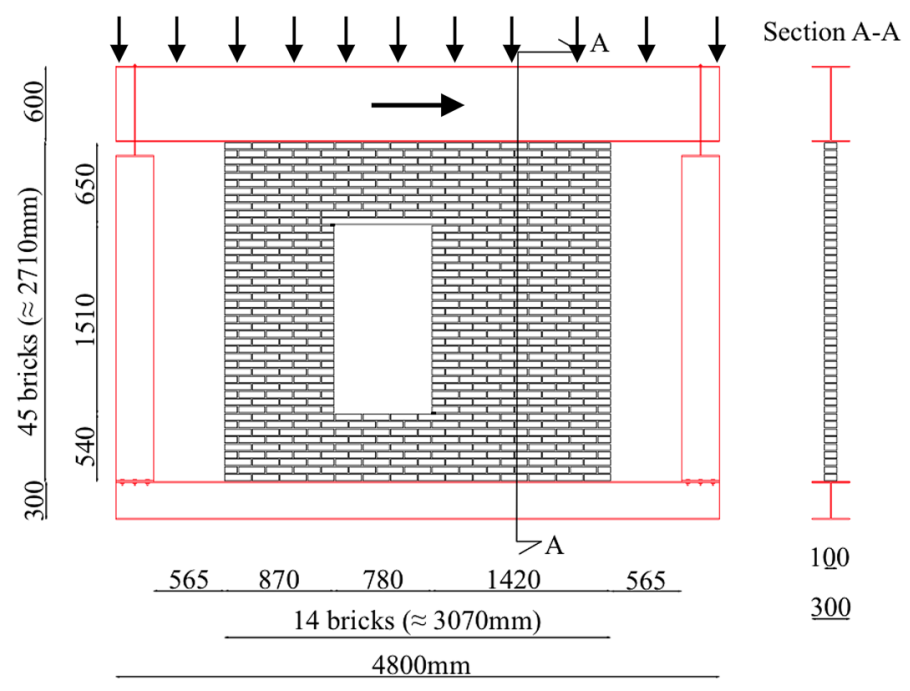

Fig. 1 Dimensions of the in-plane solid clay-brick wall with asymmetric window opening 
hysteresis. Here, the relevant model properties to achieve a reasonable fit to the experiments are described.

Finally, Sect. 6 presents the main observations and conclusions of this study.

\section{Definition and quantification of light damage}

Within the formulation of damage states or damage grades as defined in the European Macroseismic Scale (e.g. Grünthal 1998), the first damage state (DS1) concerns aesthetic damage. Precisely, since the focus of this study is on mostly minor, visible damage, cracking has been selected as a measure (and expression) of light damage for masonry walls.

Cracks indicate a permanent and visible loss of cohesion. Two other forms of 'damage' are permanent deformations without loss of cohesion and permanent translation or rotations of the object such as tilting or uniform settlement. These two forms play a minor role for the masonry cases considered and are thus not included herein. Additionally, cracks not only express damage due to earthquakes, but also due to other potential damage such as damage due to restrained shrinkage or (differential) settlements. As such, it serves as a good parameter with which to assess the progression and accumulation of damage (Korswagen et al. 2019).

A new, dimensionless, damage level parameter $\left(\Psi_{\mathrm{D}}\right.$ or $\left.\Psi\right)$ is herein proposed. The parameter expresses the total of visible cracks in one number such that the narrowest visible cracks with a width of $0.1 \mathrm{~mm}$ result in a value of around one $(\Psi=1)$, slightly larger cracks of close to $1 \mathrm{~mm}$ width correspond to two $(\Psi=2)$ and cracks of approximately $4 \mathrm{~mm}$ in width give a value of three $(\Psi=3)$. This is drawn later in Fig. 2 .

This range has been defined as DS1 and was adapted, from the work of Boscardin and Cording (1989), Burland and Wroth (1974), and, at its latest, Giardina et al. (2013a, b), where these damage levels were based on the ease of repair of the damage (see Table 1). The $\Psi$ parameter describes these qualitative and approximate descriptions in a quantitative and reproducible manner.

The decision to categorise the initiation of light damage from a crack width of $0.1 \mathrm{~mm}$ upwards is related to the detectability of cracks. From the assessment of laboratory specimens and inspection of real-world damage cases, cracks narrower than $0.1 \mathrm{~mm}$ were difficult to see with the naked eye. In fact, from an anatomical perspective, the normal human eye can detect differences of down to $30 \mu \mathrm{m}$ in ideal light and contrast conditions (see for instance, Österberg 1935). Since cracks in masonry walls do not satisfy these ideal conditions even during rigorous inspections of plastered walls, a limit of $100 \mu \mathrm{m}$ was deemed reasonable, especially considering that the outer walls in Groningen masonry are mainly unplastered; and, since DS1 is related to aesthetic damage, damage that cannot be observed, is thus not relevant. This boundary is also employed as a cut-off value when measuring the length of cracks.

Equation (1) presents the new proposed parameter which includes the number of cracks, the crack width, and the crack length with a mathematical expression that objectively quantifies damage:

$$
\Psi=2 \cdot \mathrm{n}_{\mathrm{c}}^{0.15} \cdot \hat{\mathrm{c}}_{\mathrm{w}}^{0.3}
$$

where $n_{c}$ is the number of cracks in the wall/specimen; $\hat{c}_{w}$ is the width-weighted and length-averaged crack width (in $\mathrm{mm}$ ) calculated with:

$$
\hat{\mathrm{c}}_{\mathrm{w}}=\frac{\sum_{\mathrm{i}-1}^{\mathrm{n}_{\mathrm{c}}} \mathrm{c}_{\mathrm{w}, \mathrm{i}}^{2} \cdot \mathrm{c}_{\mathrm{L}, \mathrm{i}}}{\sum_{\mathrm{i}-1}^{\mathrm{n}_{\mathrm{c}}} \mathrm{c}_{\mathrm{w}, \mathrm{i}} \cdot \mathrm{c}_{\mathrm{L}, \mathrm{i}}}
$$




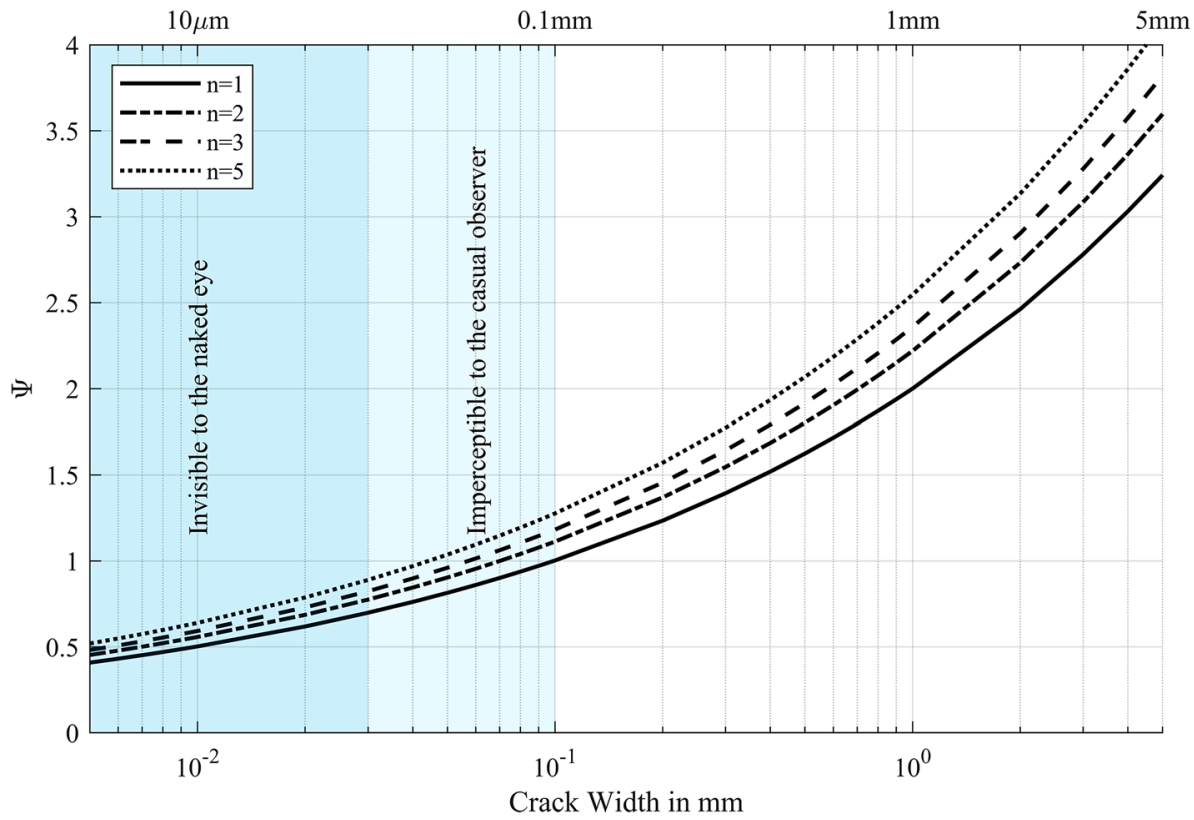

Fig. 2 Graph showing the relationship of Psi $(\Psi)$ for a given crack width according to Eq. 1. In this graph, for multiple cracks $(n>1)$ it is assumed that all cracks are of the same width. Cracks below $0.1 \mathrm{~mm}$ require special attention to be detected

where $c_{w}$ is the maximum crack width along each crack in $m m ; c_{L}$ is the crack length in $\mathrm{mm}$.

For $\mathrm{n}_{\mathrm{c}}=1, \hat{\mathrm{c}}_{\mathrm{w}}=\mathrm{c}_{\mathrm{w}}$. In this expression, the crack width of each crack is measured at their widest point.

The parameter equation is graphically shown in Fig. 2 for some values of ' $n_{c}$ '. The exponents $(0.15$ and 0.30$)$ and coefficient (2) in the expression (Eq. 1) are tuned such that the relationship to the damage levels shown in Table 1 is maintained. Since these are qualitative descriptions, the defining expression of $\Psi$ can be made to fit nicely. A summary of the relation between psi and the approximate crack width for the various damage levels given these exponents ( 0.15 and 0.30$)$ and the coefficient (2) is presented in Table 2.

The $\Psi$ parameter allows for the comparison of the intensity of damage regardless of the specimen size. This is in-line with the parent damage scale (DS1-DS5), where the damage states are independent of the size of the structure and only the importance of the damage to each specific structure is considered. This is particularly advantageous when observing the progression of damage, and comparing it between samples of different dimensions.

Furthermore, since $\Psi$ is related to the ease of repair of the damage, when the parameter is multiplied by the area of the affected wall, then a direct relationship to the cost of the repair can be obtained. This and the assemblage of multiple walls is not treated in this paper, but can be found in Appendix 1, together with a summary of the equations pertaining to the damage parameter.

For this paper, the use of this single parameter allows for the direct damage evaluation of computational results using finite elements, and of laboratory experiments using Digital Image Correlation (DIC) (Fig. 3). Since damage is directly evaluated independently of the crack configuration, the progress (or intensification) of damage can be 


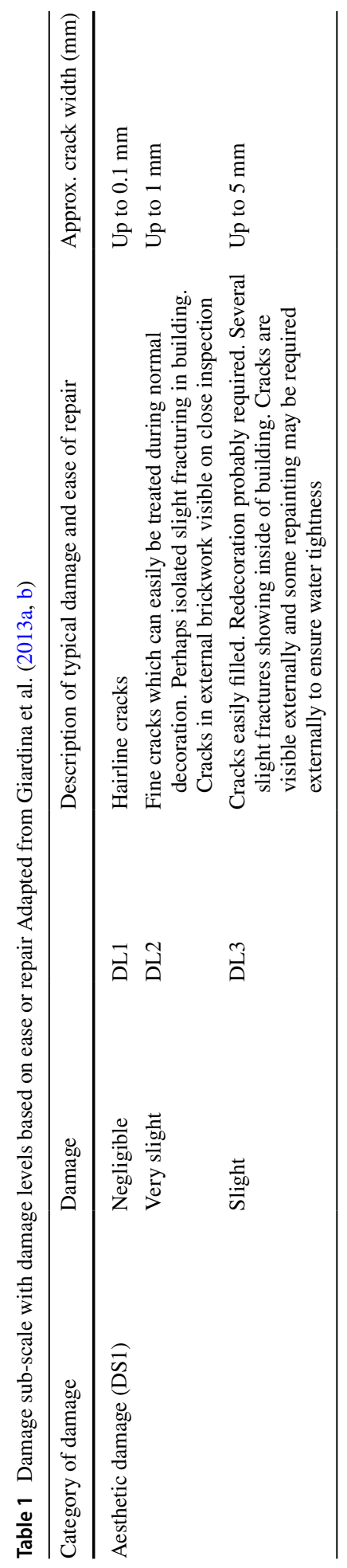


Table 2 Discretisation of the damage parameter in damage sub-levels

\begin{tabular}{llllll}
\hline Damage state & DS0 & DS1 & & DS2 \\
\cline { 3 - 5 } Damage level & DL0 & DL1 & DL2 & DL3 & DL4 \\
\hline $\begin{array}{l}\text { Parameter of damage } \\
\text { Aproximate crack width }\end{array}$ & $\begin{array}{r}\Psi_{\mathrm{D}}<1 \\
\begin{array}{r}\text { Impercepti- } \\
\text { ble cracks }\end{array}\end{array}$ & $\begin{array}{l}1<\Psi_{\mathrm{D}}<1.4 \\
\text { Up to } 0.1 \mathrm{~mm}\end{array}$ & $\begin{array}{l}1.5<\Psi_{\mathrm{D}}<2.4 \\
\text { Up to } 1 \mathrm{~mm}\end{array}$ & $\begin{array}{l}2.5<\Psi_{\mathrm{D}}<3.4 \\
\text { Up to } 5 \mathrm{~mm}\end{array}$ & $\begin{array}{l}\Psi_{\mathrm{D}}>3.5 \\
5-15 \mathrm{~mm}\end{array}$ \\
\hline
\end{tabular}
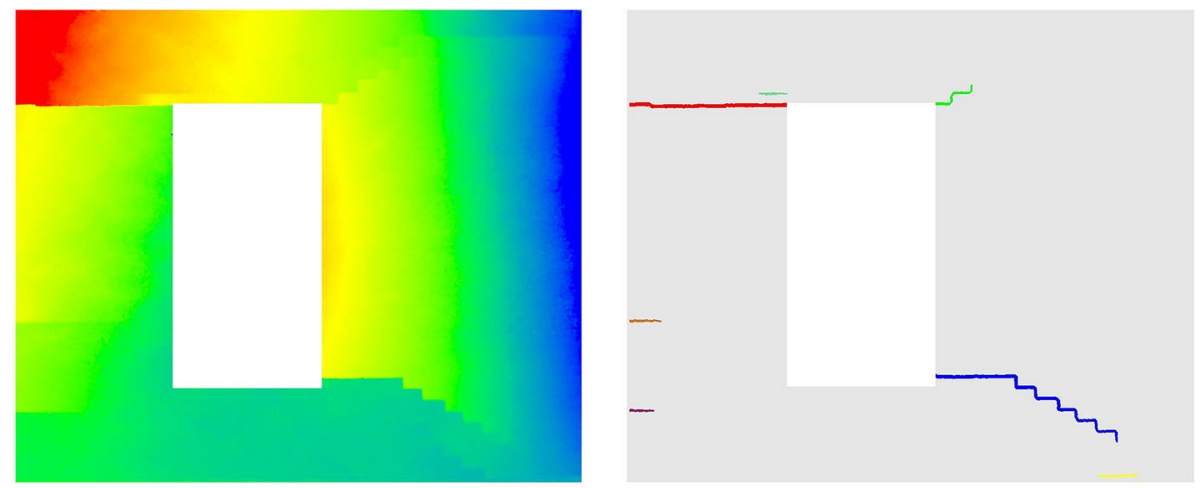

$$
\begin{gathered}
\frac{1^{2} \cdot 860+0.47^{2} \cdot 1100+0.17^{2} \cdot 250+0.16^{2} \cdot 180+0.14^{2} \cdot 140+0.13^{2} \cdot 150+0.11^{2} \cdot 210}{1 \cdot 860+0.47 \cdot 1100+0.17 \cdot 250+0.16 \cdot 180+0.14 \cdot 140+0.13 \cdot 150+0.11 \cdot 210}=0.75 \\
2 \cdot 7^{0.15} \cdot 0.75^{0.3}=2.5
\end{gathered}
$$

Fig. 3 Examples of filled contour plots of vertical displacements (left) and detected cracks (right) obtained from a DIC analysis of a laboratory wall. The maximum crack width is $1 \mathrm{~mm}$ and only cracks above $0.1 \mathrm{~mm}$ are shown, the computation of the $\Psi$ parameter is also detailed. $\Psi=2.5$

observed throughout experiments. Nevertheless, the use of one parameter to characterise the entire damage picture of a specimen is accompanied by certain limitations.

First, there is the loss of cause as the mechanism observed in a crack pattern cannot be captured in the value of one parameter. Second, in some cases there is a loss of veridicality: some changes such as the increase in length of one narrow crack while observing no changes in any other cracks, will produce an unexpected change in the value of $\Psi$. This is an unrealistic situation for which the parameter has not been calibrated. Such changes, however, have a small influence in the value of Psi and will be limited to a centesimal change. This leads to a loss of precision, but helps to realise that attempting to capture aesthetic damage with a high precision is not sensible.

In this light, the parameter needs always to be evaluated within realistic scenarios. For example, masonry walls are subjected to a limited number of cracks: attempting to evaluate $\Psi$ with a high number of cracks is hence unrealistic. Moreover, since the parameter is related to the ease of repair, which in turn is related to the width of the cracks and not to their length, as was shown in Table 1, an extension in crack length will not necessarily lead to an increase in $\Psi$; in masonry, a significant increase in length is accompanied by a realistic increase in width, which is then reflected by a higher $\Psi$ value. 
In sum, the advantages of using a single parameter, such as: inter-specimen comparability, clearer monitoring of damage progression, automation, laboratory-computational comparability, quantifiability, objectivity, and quick depiction of the damage condition, outweigh the limitations and make the parameter especially suited for the crack monitoring conducted in this study.

\section{Laboratory test program for light damage walls and window banks (spandrels)}

This section presents the laboratory tests performed in this study, see Table 3 . The reasoning for each test and its loading protocol is argued here. The results and their discussion are then presented in the following chapter.

\subsection{Material selection}

The material of all the specimens in this study was replicated, clay-brick masonry typical of the period before 1950 in the Netherlands. Jafari et al. (2017) conducted a comprehensive campaign to characterise Dutch Masonry using samples from existing or demolished structures, as well as replicated masonry. The campaign involved compression tests, tripletshear tests, bond-wrench tests, in-plane bending tests, and two out-of-plane bending tests.

Based on this study, baked-clay bricks of $210 \times 50 \times 100 \mathrm{~mm}$ of type Euroa vb WF were selected. The mortar was acquired pre-mixed with a recipe consisting of cement and hydrated lime as cementitious components, and fine sand. The final product had a ratio in weight of approximately 1.3:5.5:1 of cementitious aggregates, sand, and water. Limited companion tests were performed to verify the compatibility of the material. The main properties of the material employed are collected in Table 7 attached at the end of the paper (see column for 2017).

All specimens were built of single-wythe, running-bond masonry to aid in the detection of cracks and to limit the complexity of the samples. The walls were built using a single-row concrete lintel with the purpose of reducing the variability introduced by more complex masonry lintels constructed with bricks, thus focusing more on the crack progression in the wall. Specimens were built in 2017 and left to harden for at least 4 weeks before being tested.

\subsection{Selection of cyclic loading protocols for light damage}

The loading protocol is essential during laboratory testing. The protocol defines, together with the boundary conditions imposed by the test setup, the stresses and strains to which the samples will be subjected. For the tests to be applicable and relevant to the goals of the project, the loading protocol needs to be adequately defined. In particular, when looking at light damage caused by earthquakes, the entire loading history is relevant.

Accordingly, the loading protocols have been designed based on the following requirements:

- The behaviour of cracks (initiation and propagation) needs to be distinguishable,

- The number of cycles and their amplitudes should be representative of the target earthquakes, 


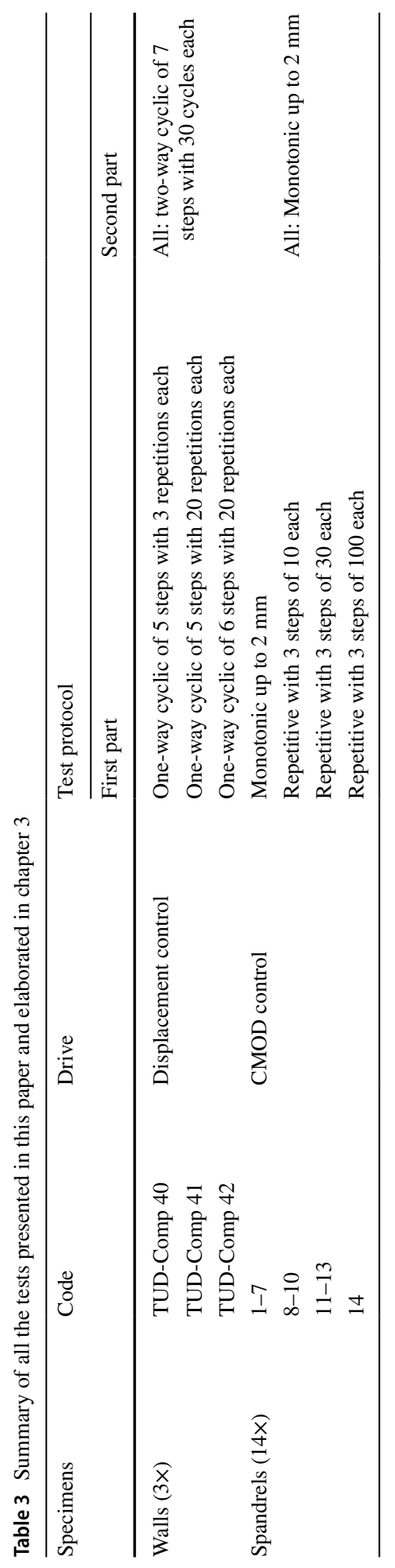


- The effect of potential degradation due to multiple earthquakes should be verifiable.

Since damaged specimens incur into the non-linear domain, the experiments are carried out under displacement-control schemes. The enforced displacement is varied throughout the test. The requirements led to the selection of repetitive loading, also known as one-way cyclic loading (positive direction in the setup), under the principle that it is important to limit the reversal of stresses on the crack surfaces for a clearer understanding of the crack progression between cycles. Then, this repetitive portion of the protocol is followed by a two-way cyclic loading (both positive and negative directions), more representative of seismic excitation. This also allows for comparison between the progression of cracks in the pre-cracked (positive) or the virgin (negative) loading directions during the cyclic portion.

Further, to monitor for potential material degradation, it was decided to apply cycles of equal displacement amplitude, so that the degradation is then evidenced by a force reduction. This means that a certain number of cycles will be identical. This leads to a step-wise incremental loading protocol.

Finally, the number of cycles and their relative amplitudes are partially based on the methodology proposed by Mergos and Beyer (2014). In Mergos and Beyer (2015), loading protocols for regions of low seismicity are discussed for various types of structures and their ductility potential. The study is based on the analysis of varied ground motion data and inspects the effective number of cycles and their amplitudes. Further, Beyer and Mergos (2015) observe that the number of cycles is more important when considering flexural (tensile driven) failure mechanisms as opposed to shear failure.

These studies are complemented with the notion that the structures to be lightly damaged are exposed to a relatively large number of earthquakes. In recent years, at least five events of magnitude greater than $\mathrm{Mw}=2.0$ have occurred yearly in the Groningen region, see for instance, Spetzler and Dost (2017). It follows that if the structures remain within the first damage state, they will have experienced a large number of small cycles.

Moreover, large-amplitude cycles have a low vibration period and are only present in larger earthquakes for ground motion data recorded close to the epicentre, observable for example in Bal et al. (2018). Note that most structures are located further from the epicentres and that flexural crack propagation occurs in masonry structures with high natural periods.

Furthermore, for lower damage states, the ratio of displacement demand and displacement capacity should be chosen to be significantly smaller than one.

Figure 4 compares the function proposed by Beyer and Mergos (2015) with the chosen loading protocols. Note that: firstly, the number of cycles has been increased in comparison to the values suggested by Beyer and Mergos (2015) under the assumption of a history of multiple earthquakes (ten equal earthquakes). Here, however, the earthquake cycles have been re-arranged to better observe the potential degradation effect. Also, Bal et al. (2018) note that while induced earthquakes do not differ significantly from tectonic earthquakes, damage accumulation may be present due to repetitive induced events.

Secondly, the initial, repetitive part of the test does not fit with the suggested protocol by Beyer and Mergos (2015). Nevertheless, the seemingly large amplitude, because it corresponds only to a displacement in one direction, is likely to lead to lower overall damage that the same amplitude in two directions, and could thus be seen as actually having a lower amplitude. Moreover, trial tests showed that the very small amplitudes that would need to be selected when following the proposed methodology precisely (Beyer and Mergos 2015) would remain in the fully-elastic region of the test specimen and would have thus been unimportant. Note that the consulted methodology includes an 'alpha' parameter 

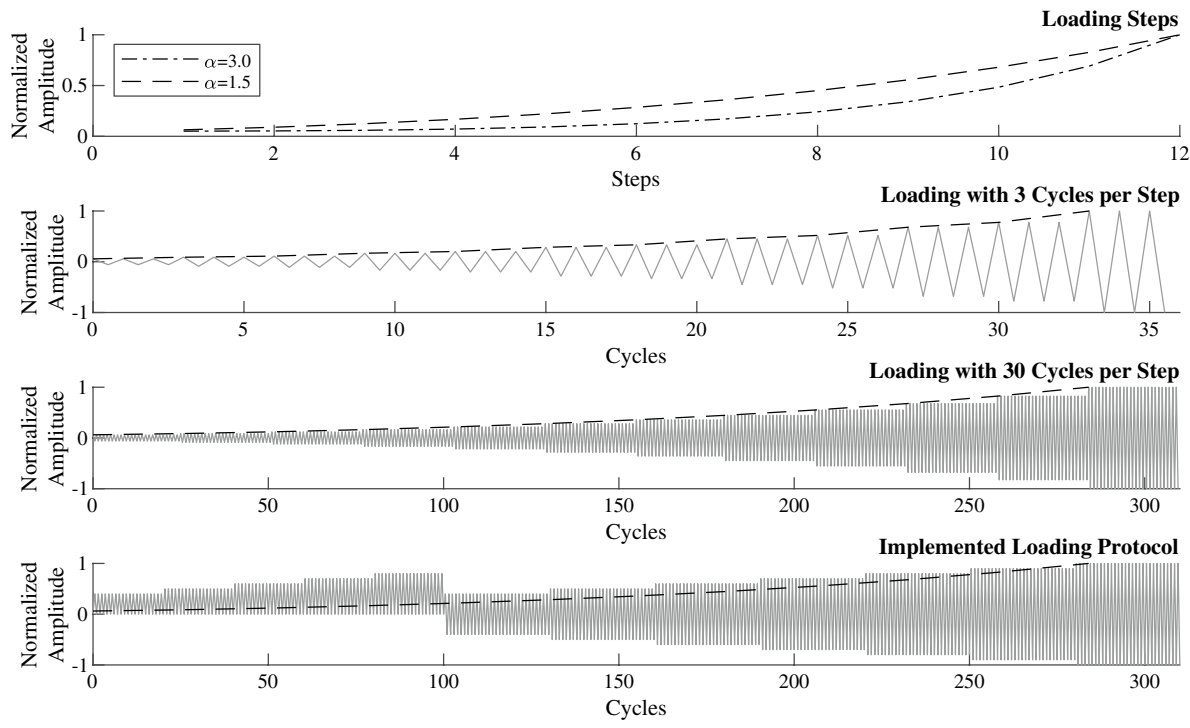

Fig. 4 Comparison of proposed loading protocols in this study with the methodology of Beyer and Mergos (2015). Top to bottom: Loading steps according to Beyer for 12 steps and two different values of 'alpha'; loading cycles according to the aforementioned methodology with 12 steps and 3 cycles per step at $\alpha=1.5$; adapted loading with 30 cycles per step; and, loading as implemented for the walls of this study consisting of a repetitive and a cyclic part with a total of 310 cycles

which relates to the natural period of the structure; in this case, the comparison is done against a value of 1.5 which corresponds to periods higher than $0.3 \mathrm{~s}$.

Consequently, when considering lightly damaged, unreinforced masonry structures exposed to a large number of small earthquakes, and the additional goals of observing the mechanics of crack propagation and potential material degradation, an incrementally repetitive loading protocol with a relatively high number of cycles, followed by an incrementally cyclic protocol has been selected, as has been shown in Fig. 4.

\subsection{Setup for lateral in-plane loading of full-scale walls}

Three specimens of a full-scale wall (Figs. 1, 5) were tested in the laboratory, first with a repetitive (one-way cyclic) quasi-static loading, later with a cyclic (two-way) loading, also quasi-statically. During the tests, a constant overburden was maintained.

The specimens were glued to a bottom and a top steel beam. The top steel beam was connected to the bottom beam by means of steel columns, but was set free to displace and rotate during the experiment (cantilever configuration). The displacement of the beam in the out-of-plane direction of the wall was restrained by the frame of the setup against which the beam could slide over Teflon sheets. The bottom beam was fully fixed to the setup frame during the experiment.

Moreover, the weight of the top beam (and some setup components) exerted a uniform, initial vertical stress (overburden) of $0.12 \mathrm{MPa}$ on the wall specimen. The top beam was attached to a hydraulic jack which applied a horizontal displacement in the plane of the wall. The displacement of the beam was monitored by the setup and 


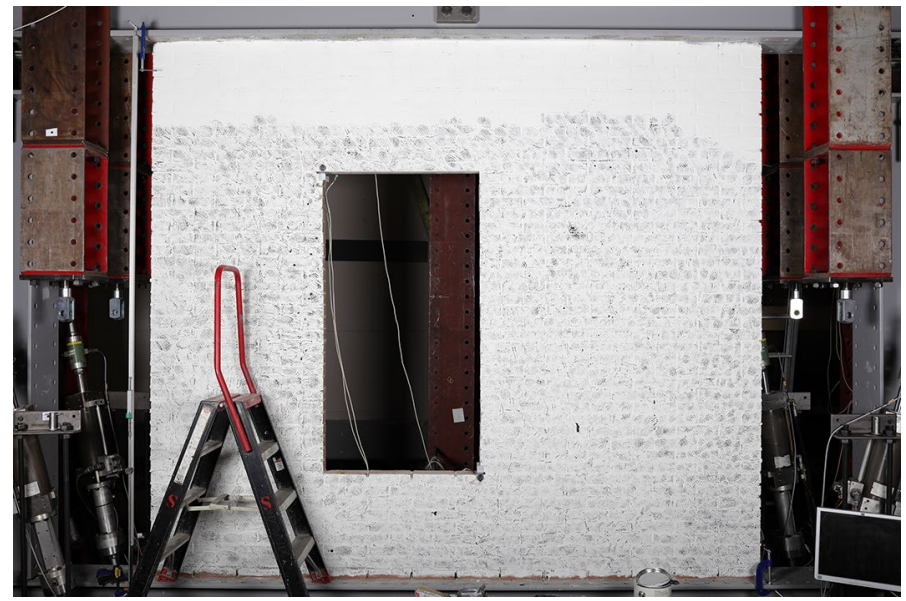

Fig. 5 Photograph of one of the experimental walls, before the application of the DIC pattern has been finished

followed the prescribed protocol. For additional details, the reader is referred to Messali et al. (2017) where the setup used to test full-scale walls in-plane is extensively presented in its various configurations.

As motivated in Sect. 3.2, the loading protocol consisted of two parts: a repetitive and a cyclic part. The first repetitive section comprised five incremental sets of equal top lateral displacement with 3 or 20 repetitions per set (step), with two walls for the latter variation. The scheme for the walls with three repetitions per step is shown in Fig. 6 . In total, three walls were tested. The first displacement amplitude corresponded to a crack width of $0.1 \mathrm{~mm}$ measured anywhere in the wall $(\Psi=1)$. Cracks were monitored locally with displacement sensors (see Esposito et al. 2016) and an overview picture of the wall was obtained with digital image correlation (see Sect. 3.5) in a manner similar to Didier et al. (2017). The crack location was estimated with a numerical prediction. Sensors were placed spanning a few rows of bricks such that they would be more likely

Fig. 6 Displacement-control scheme of the top beam lateral displacement: repetitive loading protocol. The first amplitude corresponds to a first crack of $0.1 \mathrm{~mm}$ measured at any of the crack-mouth opening sensors. Protocol shown for 5 steps of 3 repetitions per set (TUD-Comp 40)

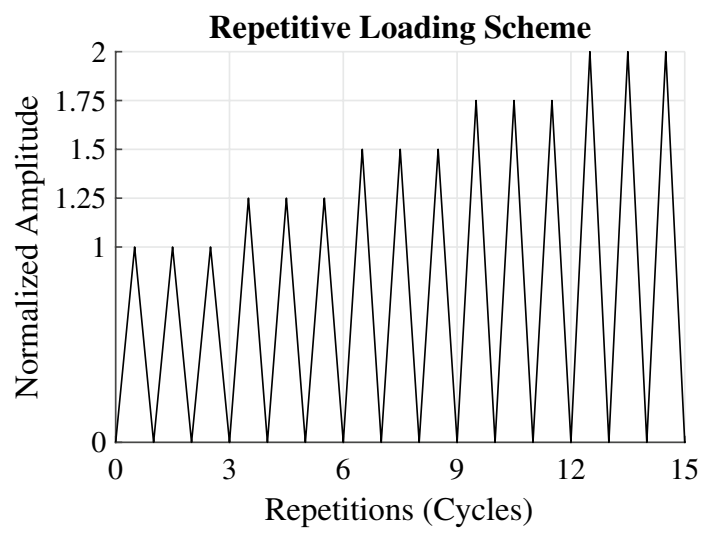


to span the actual crack locations. Subsequent steps consisted of an increase of $25 \%$ of the first displacement up to $200 \%$.

The second section of the protocol comprised cyclically applied top displacements in seven sets of 30 cycles (Fig. 7). Preliminary repetitive tests showed that the observed degradation stabilised after approximately 30 repetitions, hence this value was chosen for all cyclic loading schemes.

The initial amplitude was the same in both directions (positive and negative) and was also the same as the initial amplitude of the repetitive portion of the test (only positive). Each set corresponded to an increase of $25 \%$ up to $250 \%$.

\subsection{Setup for spandrel test (modified 4-point-bending)}

Complementary to the wall, laboratory experiments included tests of the spandrels below window banks (Figs. 8, 9). These had the goal of closely monitoring the propagation of vertical cracks in bending, and were performed monotonically and repetitively. Opposite to the work of Gattesco et al. (2016), where the behaviour of a masonry spandrel located between piers on upper floors is investigated for typical spandrel behaviour during earthquake loading (double bending and predominantly shear), the window bank tests presented here are to mimic the behaviour of a spandrel on a ground floor which may have experienced bending cracks due to actions such as settlements or restrained shrinkage. It is the repetitive effect of subsequent seismic excitations on these types of crack which is here of interest. Furthermore, this configuration is used to calibrate models to the behaviour of vertical cracks in masonry which were not present in the experimental full-scale walls as these exhibited mainly horizontal bed-joint cracks and diagonal stepped cracks, but might have appeared under different boundary conditions.

The window bank or spandrel test presented here is essentially a four-point-bending test where tension is exerted at the top of the sample, which, coupled with counterweights, allows the setup to be capable of producing cyclic loads. The counterweights are placed opposite to two hydraulic jacks (see Figs. 8, 9) and help provide a restitutive

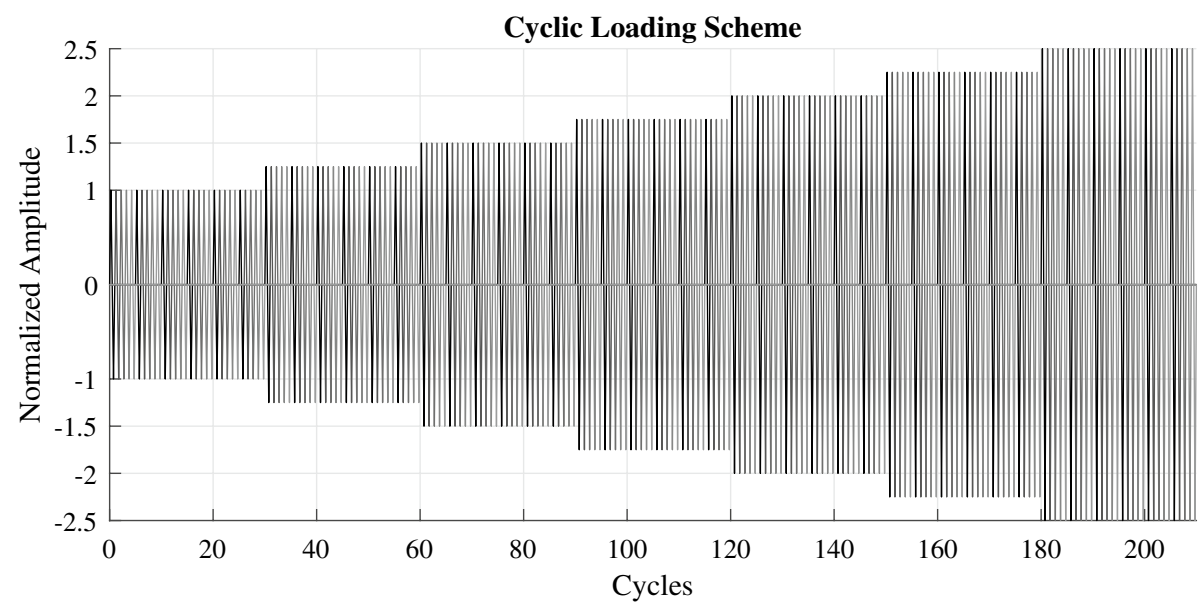

Fig. 7 Displacement-control scheme of the top beam lateral displacement: cyclic loading protocol with seven steps of 30 cycles each (210 total). The first displacement amplitude corresponds to a crack of $0.1 \mathrm{~mm}$ in width measured at any of the crack-mouth opening sensors 

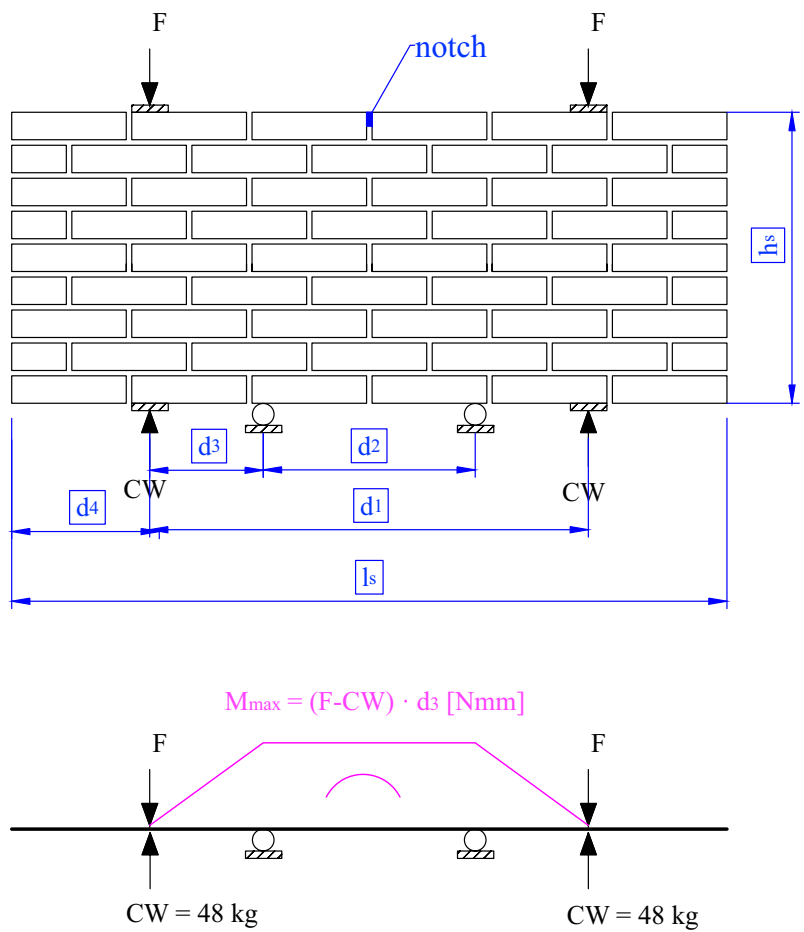

Fig. 8 Configuration of the spandrel tests with four point loading and counterweights (CW)

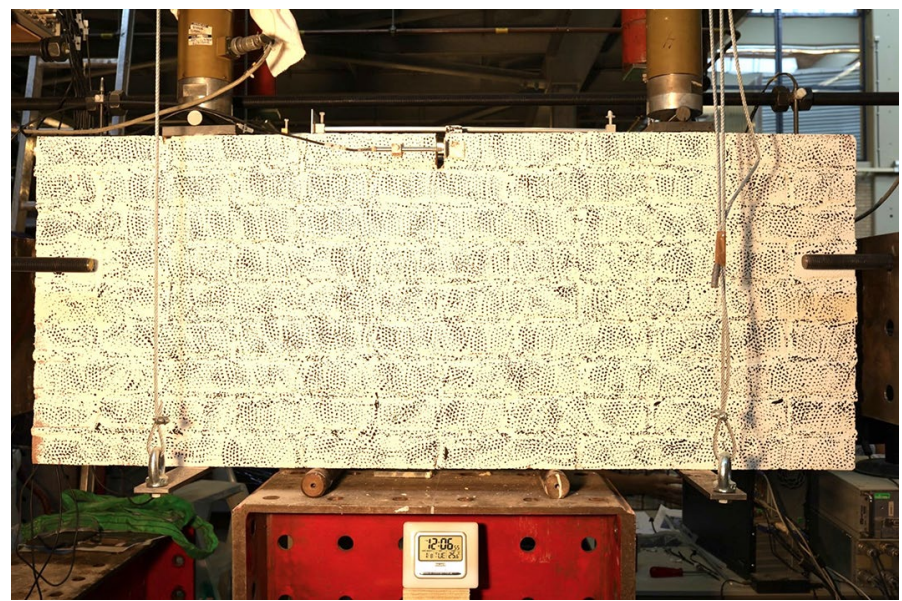

Fig. 9 Photo of a 'window bank' specimen in the setup. Painted with a pattern apt for DIC

force, as pulling the sample with the jacks would lead to tensile stresses with the risk of causing cracks in the bed joints.

First, the jacks are lowered onto the sample until a force of $1 \mathrm{kN}$ is reached, then, the counterweights are positioned and the test can begin. Consequently, during the test, 
when applying displacement repetitions, the force on each jack is never reduced below $1 \mathrm{kN}$ (see later Fig. 19) in order to maintain stability in the system.

To trigger the start of the crack in the centre of the sample, a notch was introduced by having left a piece of PVC in the central top head joint at the time of construction (see Fig. 9). However, the initiation of the crack proved to be unrelated to the existence or position of the notch, with some cracks starting on the neighbouring top head joints. Having foreseen this possibility, the experiment was conducted by monitoring the horizontal displacement over the three top head joints; i.e. the crack mouth opening displacement is used as the steering parameter of the test.

Moreover, rollers were used at every point of force application to minimise unwanted (frictional) forces. The sample was free to rotate and displace in-plane within a certain safety margin exerted by the setup. Following the notation of Fig. 8, the specimens were six bricks in width $\left(l_{\mathrm{s}}=1.3 \mathrm{~m}\right)$ and nine bricks in height $\left(\mathrm{h}_{\mathrm{s}}=0.53 \mathrm{~m}\right)$. The jacks were spaced approximately $0.78 \mathrm{~m}\left(\mathrm{~d}_{1}\right)$, and the specimens were supported at the middle with two rollers spaced $0.38 \mathrm{~m}\left(\mathrm{~d}_{2}\right)$.

Fourteen spandrel tests were performed of which seven were monotonic and the remaining were repetitive with three sets of incremental displacements (Fig. 10). Three specimens were tested with 10 repetitions per set, three with 30, and one with 100 repetitions. The amplitude of the steps was determined based on the results of the monotonic tests such that they occurred in the non-linear region before the peak force. All tests were controlled based on the CMOD measured at the top of the specimens.

\subsection{Digital image correlation for crack monitoring}

In laboratory environments, the measurement of forces and displacements is essential. Digital Image Correlation is a technique which allows the surveying of displacements. As its name suggests, DIC utilises digital imagery to obtain a displacement field from the specimen. This is accomplished by correlating two images to each other and determining the differential displacements between them.

DIC has two main advantages over traditional sensors placed on the specimens: first, it is a non-contact measuring technique which reduces the risk of the equipment being damaged and limits the interference of measuring equipment on the sample.

Fig. 10 Loading scheme for repetitive spandrel tests. Case for 10 repetitions per step

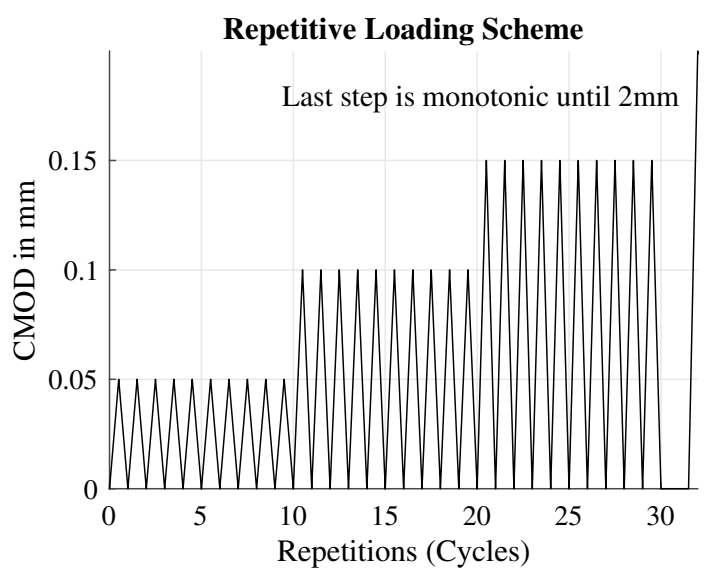


Second, it is capable of reproducing the entire field of displacements of the sample; for the walls in this study, approximately one million points were tracked on their surface simultaneously.

This technique can be applied from the very small scale (e.g. Laurin et al. 2012) to the large scale in three dimensions (e.g. Ramos et al. 2015). The algorithm for correlation and the speckle pattern on the wall have been optimised for different applications (e.g. Crammond et al. 2013) and different methods. In masonry testing, DIC has been successfully used to evidence cracks (e.g. Mojsilović and Salmanpour 2016).

The implementation used for this study was developed in-house with the goal of maximising the resolution of the displacement field (the number of tracked points on the specimen), attaining clear discontinuities in the displacement field (cracks), and doing so at a reasonable computation time and with simple photographic equipment.

The full-scale wall was surveyed with a $51 \mathrm{MP}$, full-frame, single-reflex digital camera with a sharp lens of $35 \mathrm{~mm}$ of focal length, such that one pixel in the image corresponded to $0.5 \mathrm{~mm}$ on the specimen. The lens was stopped down to f/8.0 and the specimen was lit with a flash at a speed of 1/63,000 of a second to limit image blur due to camera shake or specimen displacement, and produce even lighting conditions. Images were captured in black-and-white at the zero and maximum displacement positions throughout the test, and at other intermediate, random points. The samples were painted in white using a brittle, matte paint, and a black paint speckle pattern was applied with negative or positive moulds.

The solution method employed to obtain displacement fields from the images made use of 'subsets', squares formed by a few pixels around the points being tracked, and considered only the rigid translation of these subsets. A limited neighbour-search algorithm was used to track the subset in subsequent images. The size of the subsets varied from $10 \times 10$ to $25 \times 25$ pixels and the search area was in the order of 4 pixels. The subsets were tracked iteratively down to displacements of 0.01 pixels.

This yielded images with up to one million correlation points which required two minutes of processing time on an 18-core desktop workstation. The displacement fields were also plotted as contour or magnified deformed-shape graphs (see Fig. 11).

For the modified four-point bending test, the same imaging setup was used, albeit replacing the flash for a standard lamp. In this case, the pattern applied on the specimen was the same as for the full-scale wall. This is a point that could be improved in future tests, as an optimised pattern is likely to produce results with greater resolution.

Additionally, the discontinuities of displacement as shown in Fig. 11, were categorised as cracks. The crack width was determined by differentiating the displacement values over the discontinuity. A crack identification algorithm was used to group neighbouring (continuous) discontinuities as single cracks and reject noise in the data. Figure 12 shows a plot of the cracks overlaid on a scheme of the wall. The same algorithm also served in a FEM context to group neighbouring integration point data into the overall notion of a 'crack'.

The setup yielded good results with an error of $25 \mu \mathrm{m}$. The error was determined by analysis of two images of zero displacement, and by comparison at joints over which traditional sensors were applied. The magnitude of the error was deemed sufficient for the analyses in this study where cracks between $0.1 \mathrm{~mm}$ and $2 \mathrm{~mm}$ were observed. Note that a crack is constituted by a large number of points, many of which are located close to the crack line, thus reducing the error on the reported crack width significantly. This is evident in Fig. 11 (bottom left) where the widest cracks are, at their widest point, narrower than $50 \mu \mathrm{m}$ yet clearly identifiable. 


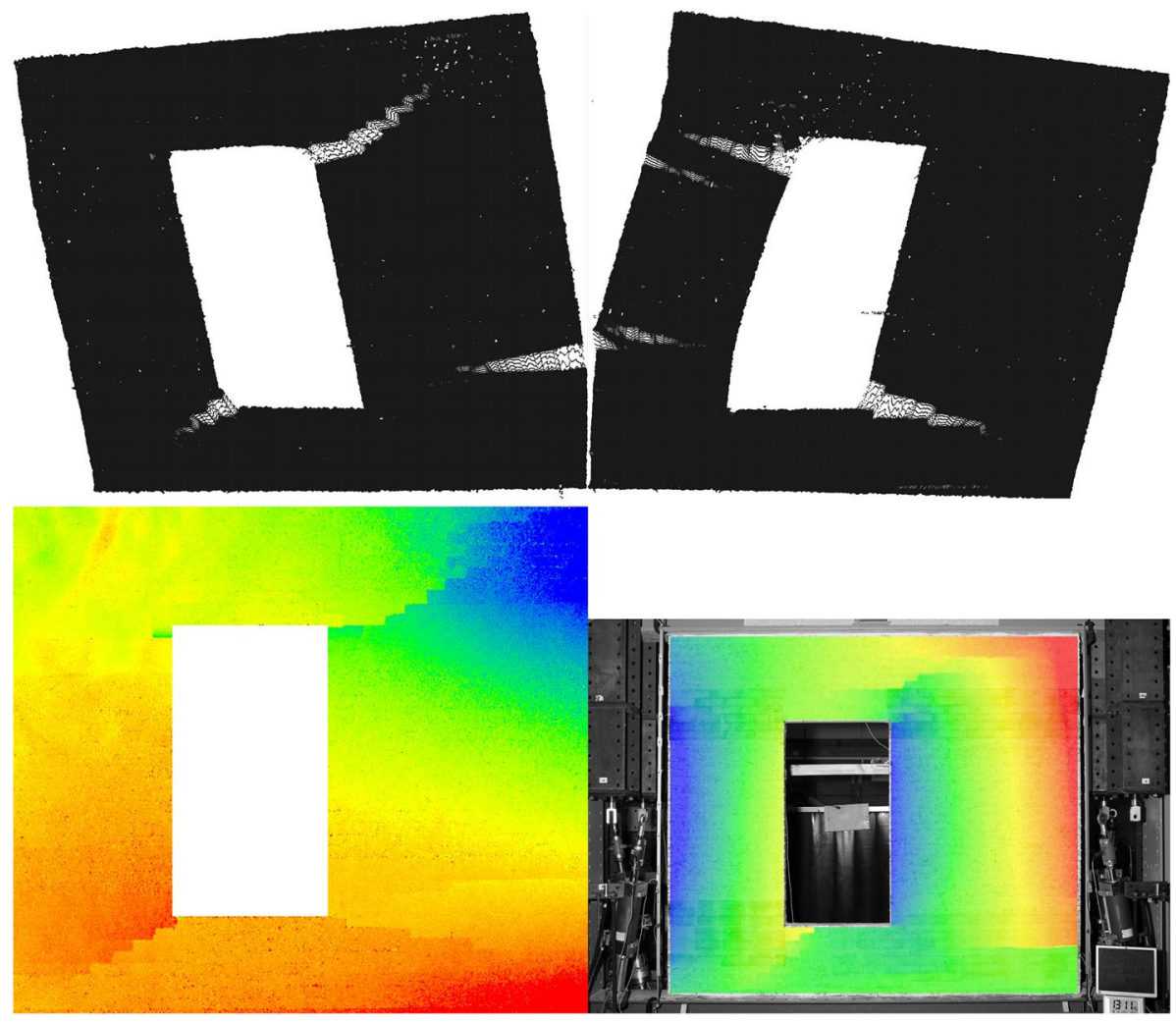

Fig. 11 Examples of different results produced with DIC. Top: deformed shape plots magnified 500 times for a lateral drift of $0.7 \%$ o. Bottom: two different specimens, horizontal and vertical contour plots, loose and overlaid, respectively. The extreme colors (red to blue) show a relative displacement of $0.45 \mathrm{~mm}$ and $1.1 \mathrm{~mm}$, at $0.1 \%$ and $0.7 \%$ of drift, respectively

Fig. 12 Crack plot showing the location of cracks and their respective width (in micrometres) and length (in millimetres) automatically post-processed from DIC measurements

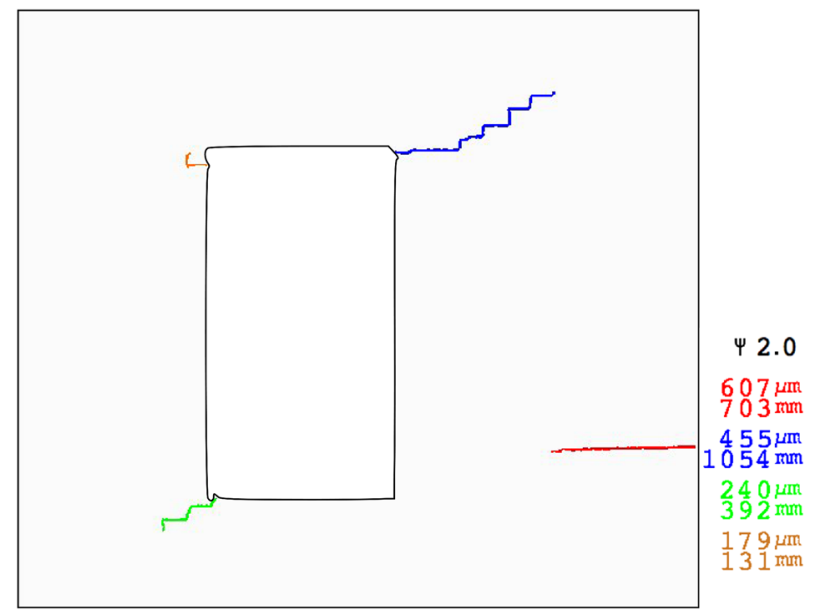




\section{Results of laboratory tests}

\subsection{Test results for full-scale walls}

During testing of the full-scale walls, cracks developed progressively up to a width of approximately $0.7 \mathrm{~mm}$ for a top lateral displacement of $2.2 \mathrm{~mm}$ in the case of one representative specimen (Fig. 13). The first crack (CMOD $\geq 0.1 \mathrm{~mm}$ ) was detected at a top displacement of $0.85 \mathrm{~mm}$ on the first wall and $0.87 \mathrm{~mm}$ for the two subsequent walls. The crack pattern differed slightly between these test cases, but consistently displayed three main cracks during the repetitive phase of the tests (see Fig. 14), and three additional cracks during the cyclic portion of the test.

It must be noted that emphasis is put on the repetitive (one-way cyclic) portion of the tests for brevity and due to the fact that computational models have been calibrated so far only with respect to the repetitive portion mainly due to the computational expense and under the premise that the walls had already been damaged in the repetitive portion of the protocol. Nonetheless, the crack progression is well monitored also throughout the cyclic portion and is therefore briefly presented next.

The evolution of the cracks focused first on rocking of the left pier by developing the cracks at the bottom left and top right of the pier. Once these cracks were fully

Fig. 13 Crack mouth opening displacement of the test with three repetitions (TUD-Comp 40). A slight increase can be observed within each incremental step (five of three repetitions each)

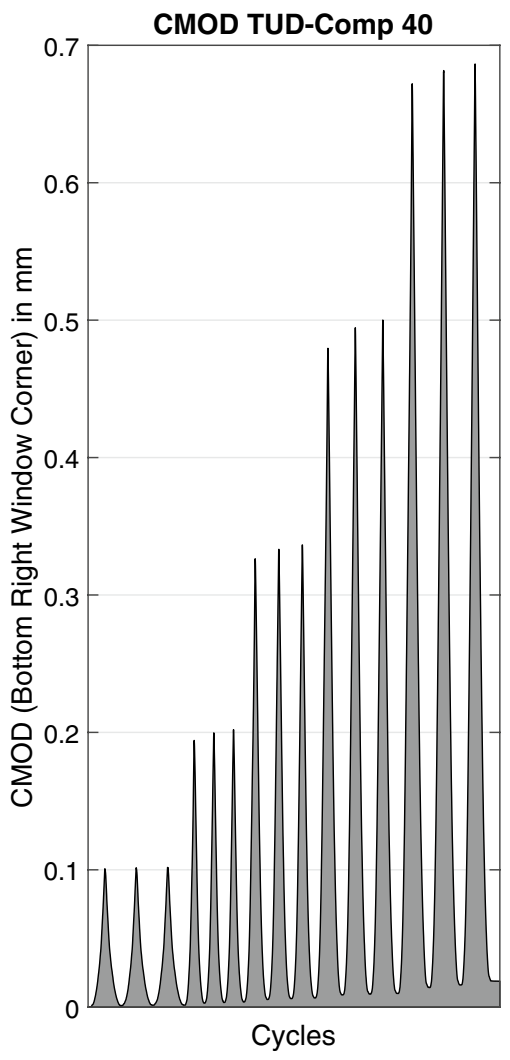




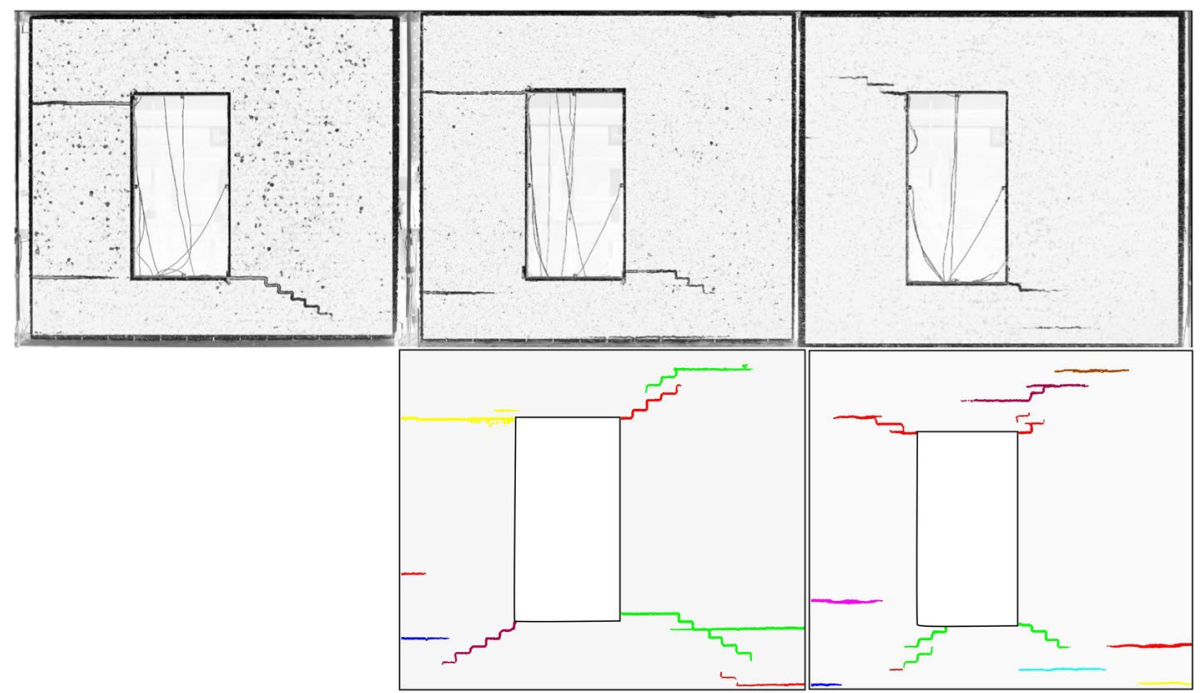

Fig. 14 Crack plots of the three full-scale wall specimens: TUD-Comp 40, TUD-Comp 41 and TUD-Comp 42 for: top, overlaid on the photo of the last step of the repetitive portion of the test, and bottom, the maximum crack history during the entire test thus including the cyclic portion. Note that cracks were not occurring simultaneously. The final picture for TUD-Comp 40 is missing due to some issues with the DIC pattern

developed (in the case of the top crack crossing the entire pier horizontally), the crack at the bottom right of the window grew significantly (see Fig. 15).

The maximum shear force measured during the three tests was $22 \mathrm{kN}$ (see Fig. 16). Here, the repetitions in each set revealed a minor, yet visible and consistent force reduction, mainly in the first repetitions of a set. The largest reduction was found in the set with the lateral displacement closest to that of the peak load capacity of the wall. In some cases, this force reduction corresponded to up to $3.5 \%$ and was also accompanied by an increase in the crack mouth opening displacement (CMOD) of up to 6\% as was shown in Fig. 13.

After analysis of DIC results for multiple values of in-plane lateral drift of the walls, a relationship between drift and the intensity of damage could be observed. In Fig. 17a, this relationship appears to be linear. To avoid the bias introduced by considering many points at values of equal drift, and to minimise the influence of the progressive damage on the specimens, only the pictures taken at random points during the repetitive test have been considered. Since it is expected that at zero drift, no damage will be present, the Theil-Sen method for regression of slopes has been used (Theil 1950). This method is more robust against outliers than fitting of a linear model with the least squares method. For the slope,
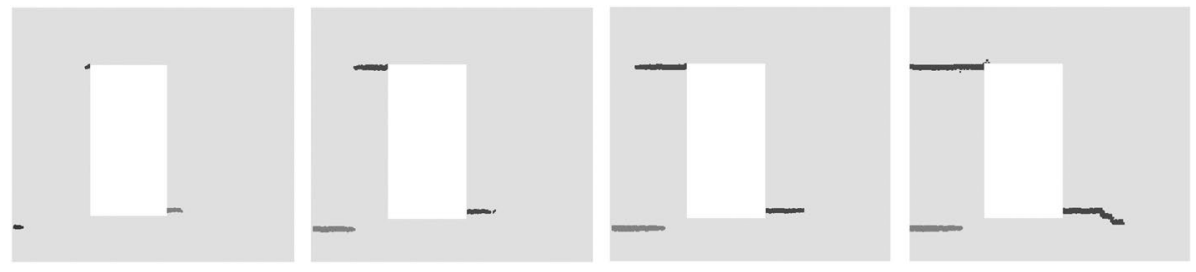

Fig. 15 Sequence of crack propagation for different steps in the repetitive test of TUD-Comp 41 

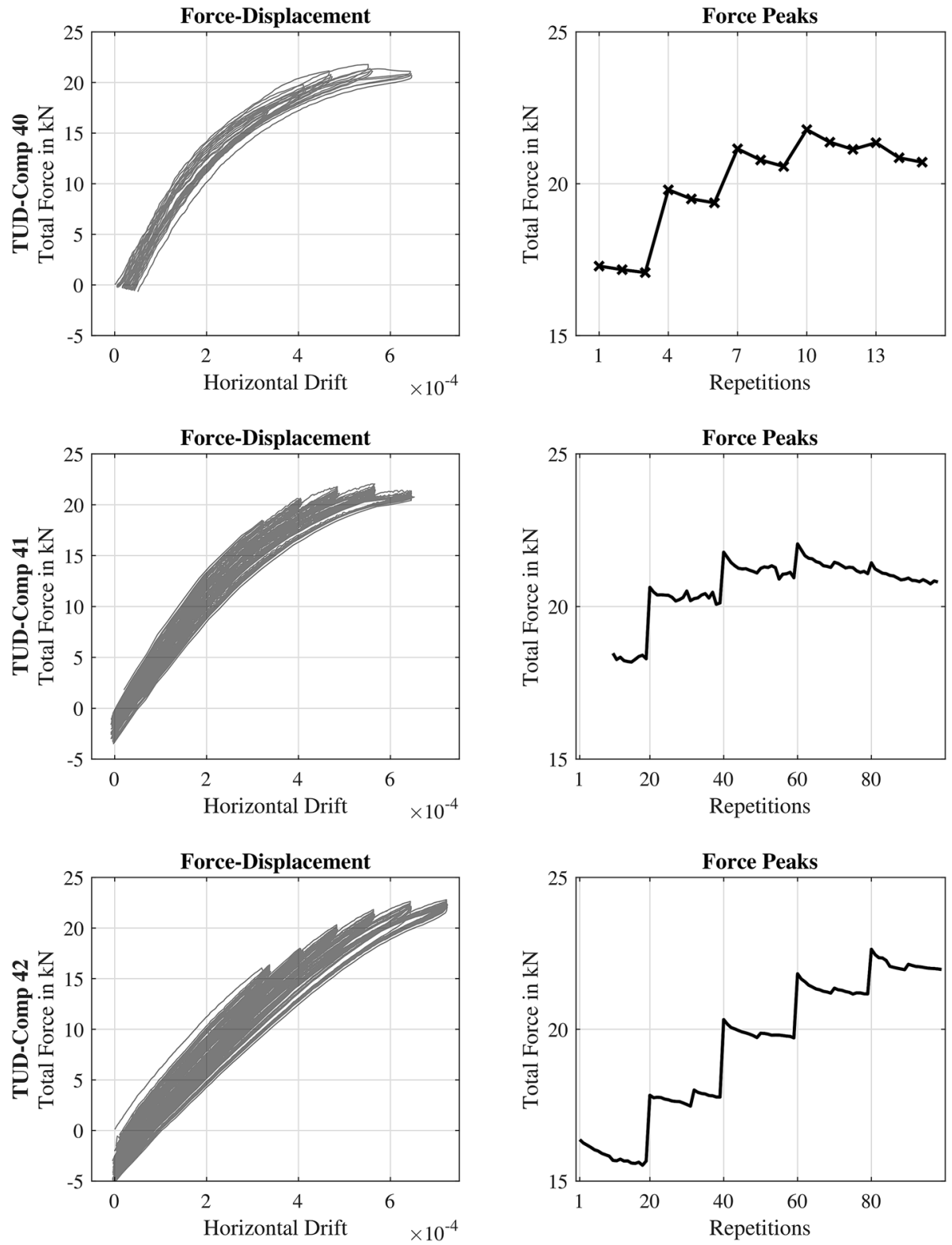

Fig. 16 Force-displacement curves for the repetitive portion of the tests of the three test walls on the left, and the corresponding force reduction observed on each step on the right (first graph includes markers)

the median of the sampled points is computed, and the standard deviation is obtained from a lognormal fit to the data.

When observing the data points obtained from the cyclic portion of the test in Fig. 17b, a linear trend not passing through from the origin can be observed and is highlighted with a linear regression line. This is expected, since the wall has experienced damage and shows 

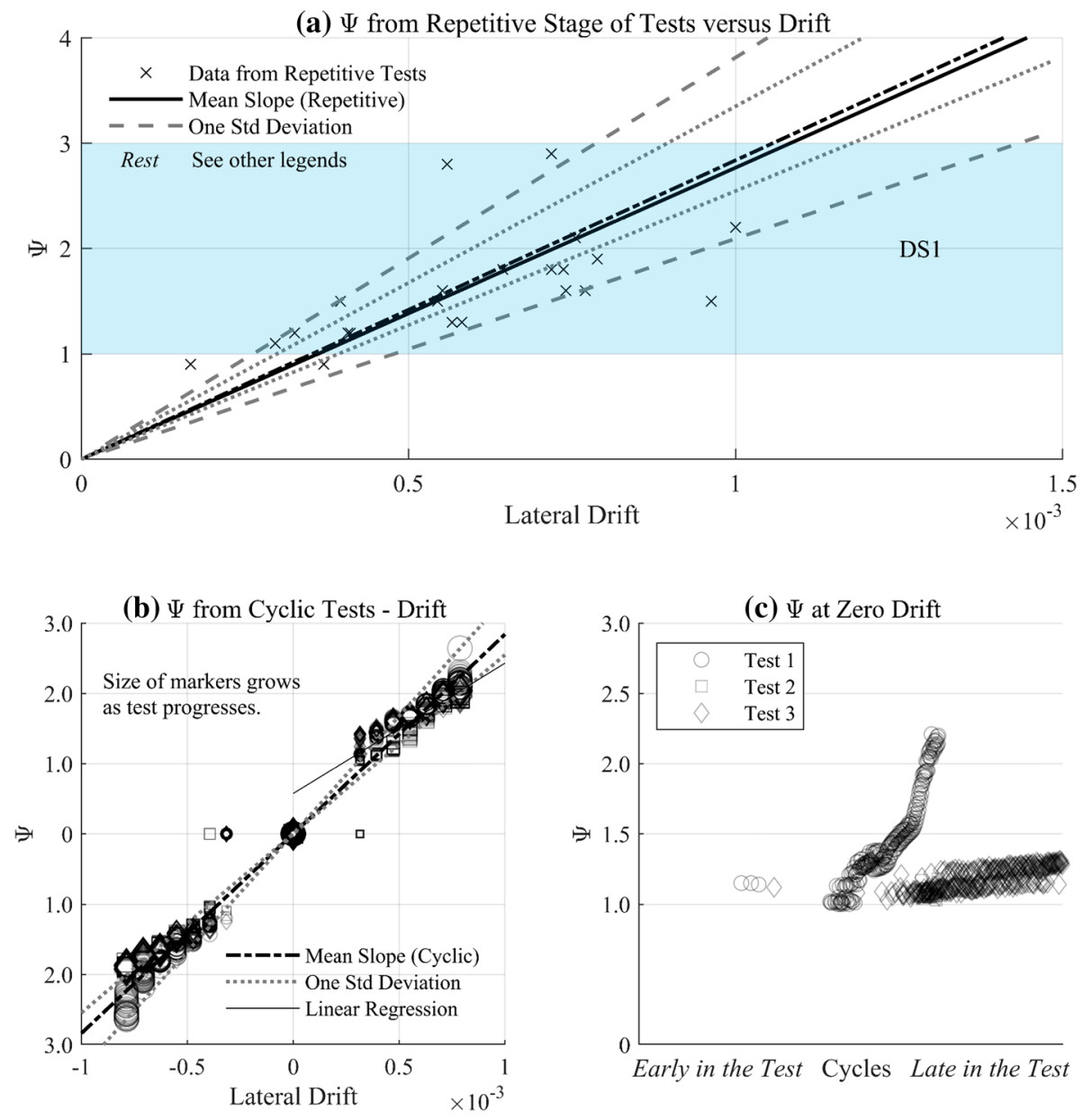

Fig. 17 a Linear regression (Theil-Sen method) of results for the damage parameter $\Psi$ against lateral drift of masonry test walls showing only random drift values during repetitive tests and highlighting the zone corresponding to damage state one (DS1, light damage). b Results from the cyclic part of the tests; and c values of Psi at zero drift due to residual damage from a high number of cycles towards the end of the test

signs of residual damage already at zero values of drift, the latter is shown in Fig. 17c. Nonetheless, fitting a slope model to all the available points yields a similar result as when only the initial, random points during the repetitive portion of the test are considered, see the dotted line in Fig. 17b which is also reproduced Fig. 17a where it appears next to the solid line. The accumulated damage also explains why the standard deviation of the fewer points is greater than that of the cyclic data.

Based on these results, it is concluded that for the geometry of the tested wall, drift values between $0.3 \%$ and $1.1 \%$ correspond to damage state one as highlighted in Fig. 17.

Furthermore, during the cyclic portion of the test, the propagation of cracks was closely monitored to determine whether a relationship with the force reduction would exist. Indeed, during the seven steps of the cyclic protocol, minor increases in the value of $\Psi$ were observed sometimes during the same step. This increase in $\Psi$ resulted from a small, 
yet consistent increase in crack width during some of the steps. An example of such development of crack width is presented in Fig. 18.

\subsection{Test results for window banks (spandrels)}

The objective of this test was to explore the cracking behaviour of masonry when cracks are propagating in a toothed vertical manner through the specimens. This failure mechanism is commonly observed, for instance, when structures have experienced differential settlements (Van Staalduinen et al. 2018). The results of the seven monotonic and the seven repetitive tests are summarised in Fig. 19. Here, results are plotted both on a linear and a logarithmic scale for the crack mouth opening displacement; this allows for a good representation of both the post- and the pre-peak behaviour. From the monotonic tests, it was determined that the value of $0.1 \mathrm{~mm}\left(10^{-1}\right)$ lied ideally in the non-linear region before the peak, hence, values of $0.05,0.1$, and $0.15 \mathrm{~mm}$ were set for the three steps of the repetitive tests.

In these repetitive 'window bank' tests, a clear force reduction was observable within a certain step; even though the same crack mouth opening displacement was achieved during an entire repetition set, the reduction in force was up to $21 \%$ on the last step (as average of the seven specimens). This is presented in Table 4 . In fact, the closer the step was to the peak strength of the specimens, the greater was the reduction.

It was also observed that the force reduction decreased exponentially with every repetition and stabilised after approximately 30 cycles, see Fig. 20.

Furthermore, the repetitive tests achieved lower ultimate displacements than the monotonic tests; the former stopped at a CMOD value of almost $1 \mathrm{~mm}$ due to an intense

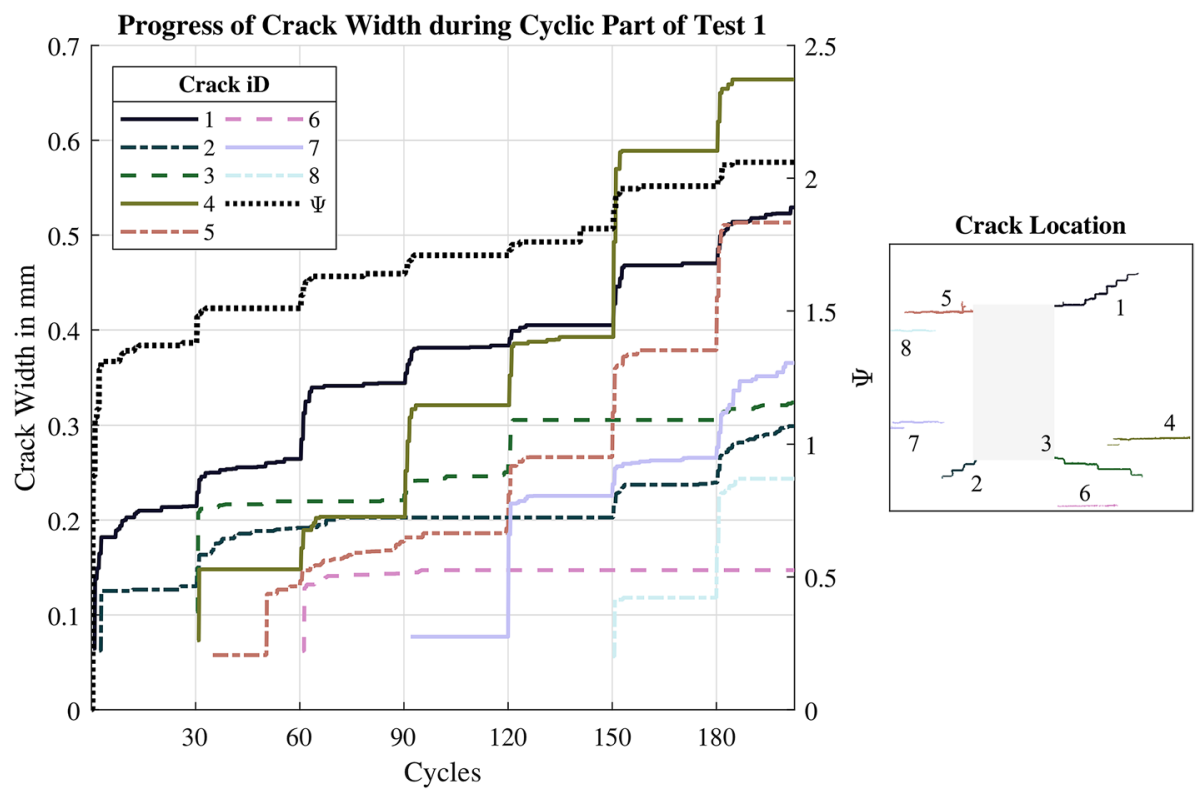

Fig. 18 Evolution of maximum crack width of various cracks on a wall during the cyclic part of the loading protocol as monitored with DIC 


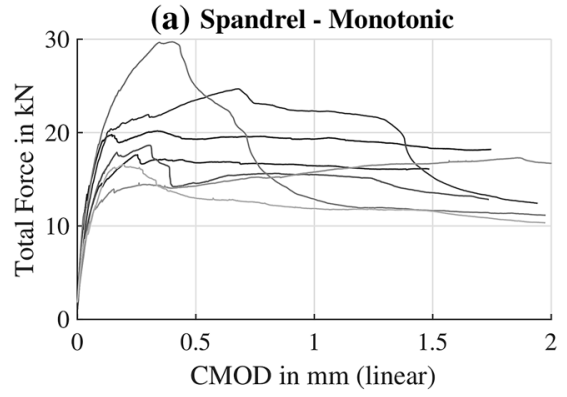

(c) Spandrel - 10 Repetitions

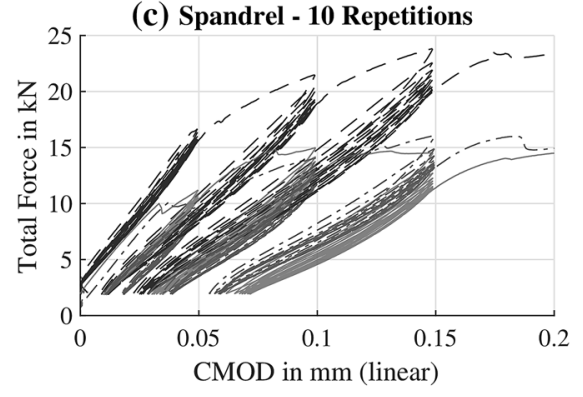

(e) Spandrel - 30 Repetitions

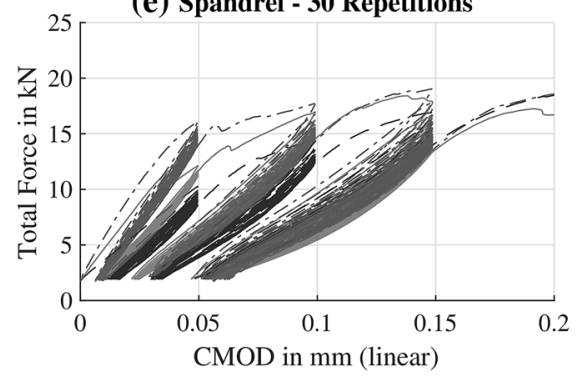

(g) Spandrel - 100 Repetitions

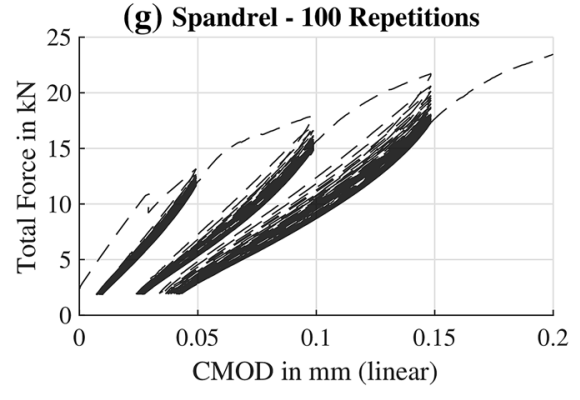

(b) Spandrel - Monotonic

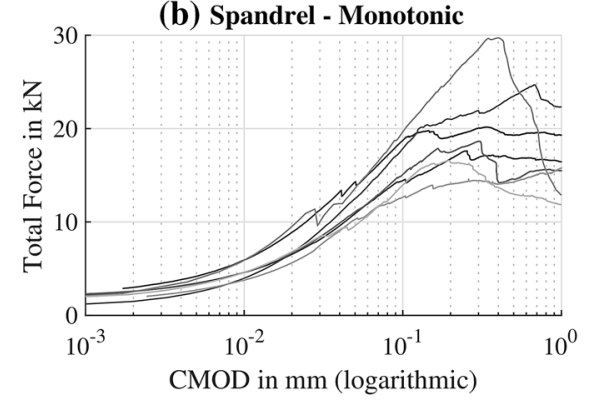

(d) Spandrel - 10 Repetitions
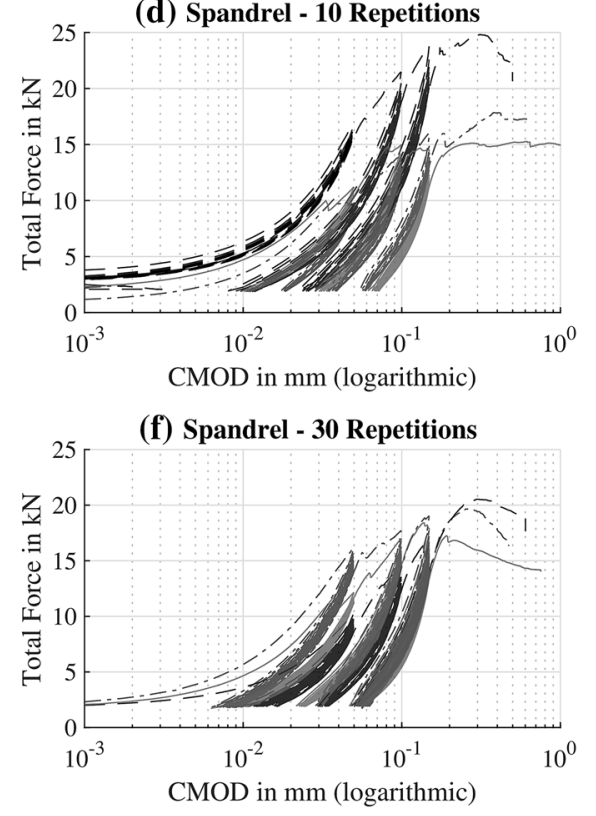

(h) Spandrel - 100 Repetitions

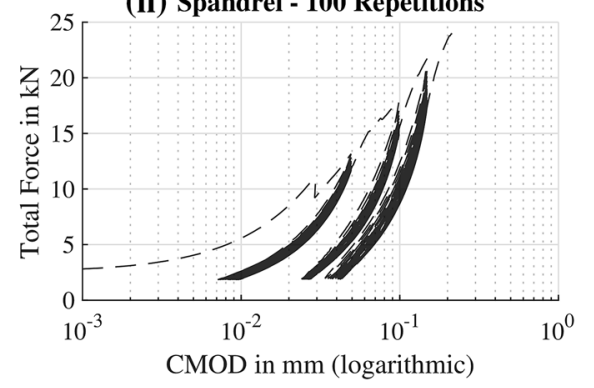

Fig. 19 Force-displacement (CMOD) curves for the 'window bank' tests, seven samples monotonically (a, b) three samples at ten $(\mathbf{c}, \mathbf{d})$, three at thirty repetitions $(\mathbf{e}, \mathbf{f})$ and one for $100(\mathbf{g}, \mathbf{h})$. The force degradation during the repetitive tests is clearly visible. Graphs are presented on the left on a linear scale, but on the right, also on a logarithmic scale to better show the development from the linear to the non-linear regime 
Table 4 Force reduction in the seven repetitive spandrel tests

\begin{tabular}{lcclll}
\hline Sample code & $\begin{array}{l}\text { Number of } \\
\text { repetitions }\end{array}$ & Step 1 (\%) & Step 2 (\%) & Step 3 (\%) & Average (\%) \\
\hline $\mathrm{N}$ & 10 & 12 & 17 & 18 & 16 \\
$\mathrm{O}$ & 10 & 8 & 17 & 23 & 16 \\
$\mathrm{U}$ & 10 & 7 & 13 & 16 & 12 \\
$\mathrm{M}$ & 30 & 4 & 14 & 27 & 15 \\
$\mathrm{R}$ & 30 & 16 & 15 & 18 & 16 \\
$\mathrm{~S}$ & 30 & 14 & 19 & 29 & 21 \\
$\mathrm{P}$ & 100 & 10 & 14 & 20 & 15 \\
Average & & 10 & 16 & 21 & \\
\hline
\end{tabular}
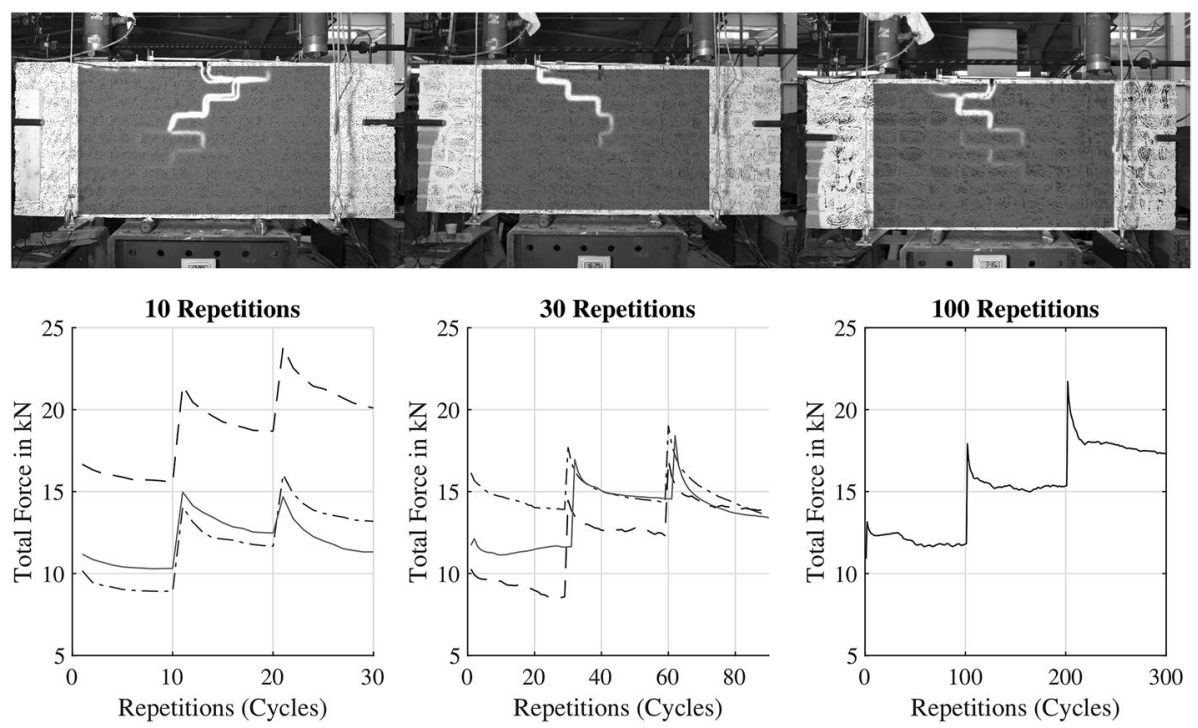

Fig. 20 Top: three different crack patterns of various spandrel tests. Bottom: maximum force achieved in each cycle for all the repetitive tests. Even though the prescribed displacement is the same, a force reduction is visible, exponentially decreasing when increasing number of cycles

reduction in force, while the latter reached values of $2 \mathrm{~mm}$ at the limit of the setup. This implies that the large amount of accumulated cycles may have reduced the toughness of the specimens. However, from the limited number of samples, no relationship can be observed between the number of repetitions and the ultimate displacement capacity of the specimens.

\subsection{Discussion and comparison between test results of walls and spandrels}

A marked contrast is observed between the smaller spandrels and the larger walls when considering the reduction in force during repetitions. While it is likely that the main causes 
for this difference are the size of the samples and the failure mechanisms, it must be noted that for the spandrels, the CMOD was controlled and repeated, while for the walls, the top lateral displacement was controlled. For the walls with top displacement control, the crack mouth opening sensors placed on the wall showed an increase of crack width of up to $6 \%$ within a repetition set. This means that, if for the walls the CMOD of one of the cracks in the wall had been kept constant during the repetitions (like for the smaller spandrels), a larger force reduction would have been observed.

Nevertheless, the degradation observed with the window bank tests is at least twice as high as that of the wall tests. This is probably due to the more dissipating failure mechanism of the window bank test, where the vertical toothed crack in between the bricks, allows for a large amount of sliding in-between repetitions. This leads to a steeper accumulation of damage. It must be noted that the DIC employed for the spandrel tests did not allow for accurate monitoring of the crack length; this is a point of improvement for future tests.

\section{Calibration of computational models}

To better understand the experimental tests, and to extrapolate results to other masonry walls and structures and to other loading configurations than what is possible in the laboratory, finite element computational models have been elaborated and calibrated to the results of the experimental tests.

Two approaches have been followed when modelling the walls: a continuum model, where bricks and mortar are homogenised into one composite material (macro modelling); and a model where the bricks and the mortar are modelled independently. In the latter case, usually zero-thickness interface elements are used between the brick and the mortar, but in the present study, continuum elements are used to represent the mortar, thus identifying this model as an adapted micro model. For additional information about the techniques used to model masonry, the reader is referred to Rots et al. (1994), Lourenço (1996) or Lourenço and Rots (1997).

The micro model is computationally more expensive but offers greater resolution when modelling cracks. Therefore, for analyses where a large number of models need to be run and the computational expense is limiting, the macro model is better suited. However, for smaller cases, like the window-bank test, where the relative size of the bricks in comparison to the sample is important, a macro model will likely produce less reliable results.

Consequently, a macro and a micro model of the full-scale walls are presented next, but for the window bank tests, only a micro model has been calibrated. All the models presented here have been elaborated in the DIANA finite element program.

\subsection{Model properties and boundaries}

This subsection details the calibrated model properties and the replicated experimental boundaries for both wall and spandrel models.

\subsubsection{Wall macro model}

Computational models that had been used to predict the behaviour of the experiments have been improved based on the experimental results to (partly) replicate the observed 
degradation and better reflect the stiffness and crack patterns. A smeared, continuum Engineering Masonry Model (EMM), recently developed by TU Delft and DIANA FEA was chosen for the elaboration of the macro (or continuum) model. This material model accounts for the orthotropy from bed and head joints, tensile softening with secant unloading, shear friction and cohesion softening with elastic unloading, and compression hardening and softening with bilinear elastic-secant unloading. For details about the material model the reader is referred to Rots et al. (2016) and Schreppers et al. (2016).

The model of the wall includes a linear-elastic concrete lintel $(\mathrm{E}=31 \mathrm{GPa})$, linear beam elements for the bottom and top beams, a fixed boundary at the bottom, and is displacement-driven at the top (see Fig. 21).

A summary of the model properties is presented next:

- Wall with opening from baked-clay brick masonry,

- Dimensions: $3.05 \times 2.70 \times 0.10 \mathrm{~m}$,

- 2D Plane Stress Model,

- Bottom (HEB300) and top (HEB600) beam modelled using beam elements,

- 8-Node Quadratic Elements,

- $3 \times 3$ Integration scheme,

- $0.12 \mathrm{MPa}$ overburden,

- Mesh size $50 \times 50 \mathrm{~mm}$,

- Full Newton-Raphson scheme with force or displacement conditions with a $1 \%$ residual tolerance for convergence.

Further calibrating the material parameters allowed for results loyal to the experiments. The employed and calibrated parameters for the material model are displayed in Table 5. Note that the EMM requires the specification of the angle at which stair-case cracks will travel diagonally in masonry, this is related to the geometry of the brick and the masonry pattern (dutch bond, english bond, etc.). Moreover, it also requires parameters relating to the compressive strength of masonry, but these did not play a role in any of the models as no compression failure was observed in the tests nor in the models.

Fig. 21 Finite element model showing mesh and boundaries

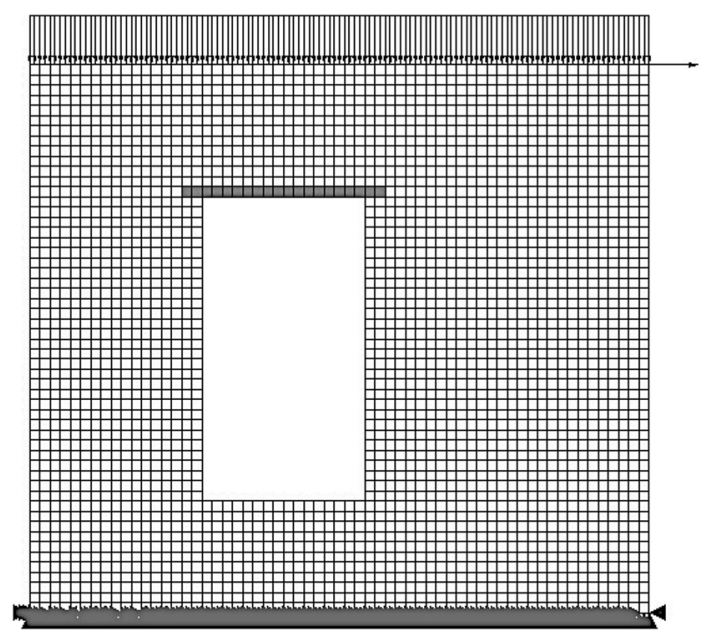


Table 5 Calibrated properties for the macro model

\begin{tabular}{llll}
\hline Property & Symbol & Value & Units \\
\hline Density & $\rho$ & $1708^{\mathrm{a}}$ & $\mathrm{kg} / \mathrm{m}^{3}$ \\
Young's modulus (vertical) & $\mathrm{E}_{\mathrm{y}}$ & $3087^{\mathrm{a}}$ & $\mathrm{MPa}$ \\
Young's modulus (horizontal) & $\mathrm{E}_{\mathrm{x}}$ & $2157^{\mathrm{b}}$ & $\mathrm{MPa}$ \\
Shear modulus & $\mathrm{G}_{\mathrm{xy}}$ & $1354^{\mathrm{b}}$ & $\mathrm{MPa}$ \\
Tensile strength & $\mathrm{f}_{\mathrm{t}}$ & $0.09^{\mathrm{a}}$ & $\mathrm{MPa}$ \\
Fracture energy (tension) & $\mathrm{G}_{\mathrm{f}}^{\mathrm{I}}$ & $7.527^{\mathrm{c}}$ & $\mathrm{N} / \mathrm{m}$ \\
Compressive strength & $\mathrm{f}_{\mathrm{c}}$ & $11.35^{\mathrm{a}}$ & $\mathrm{MPa}$ \\
Fracture energy (Compression) & $\mathrm{G}_{\mathrm{C}}$ & $26050^{\mathrm{a}}$ & $\mathrm{N} / \mathrm{m}$ \\
Friction angle & $\varphi$ & $0.669^{\mathrm{a}}$ & $\mathrm{rad}$ \\
Cohesion (no softening) & $\mathrm{c}$ & $0.14^{\mathrm{a}}$ & $\mathrm{MPa}$ \\
Diagonal crack orientation & $\alpha$ & $0.5^{\mathrm{a}}$ & $\mathrm{rad}$ \\
\hline
\end{tabular}

${ }^{\mathrm{a}}$ From companion tests (material characterisation)

${ }^{\mathrm{b}}$ From calibration

${ }^{c}$ From formulation (Schreppers et al. 2016)

\subsubsection{Wall micro model}

The micro model differs from the macro model in that bricks and mortar are modelled independently, each with their own material properties (see Fig. 22). Since no damage was observed in bricks, these are modelled linear-elastically, significantly improving the computation time of the model and its simplicity. Moreover, since no interface is used between the bricks and the mortar, all non-linear properties are gathered in the mortar; here, the strength of the bond between brick and mortar is used as the strength of the mortar. Furthermore, it is possible to differentiate between the mortar in the head joints and that in the bed joints. The former being of lower quality (or strength) due to the construction practice where head joints are filled in-between bricks, while bed joints are pressed with the bricks above it. Since the mortar is a homogenous material, a total strain, rotating crack model was used; as its name suggests, this model has a single direction for a stress-strain

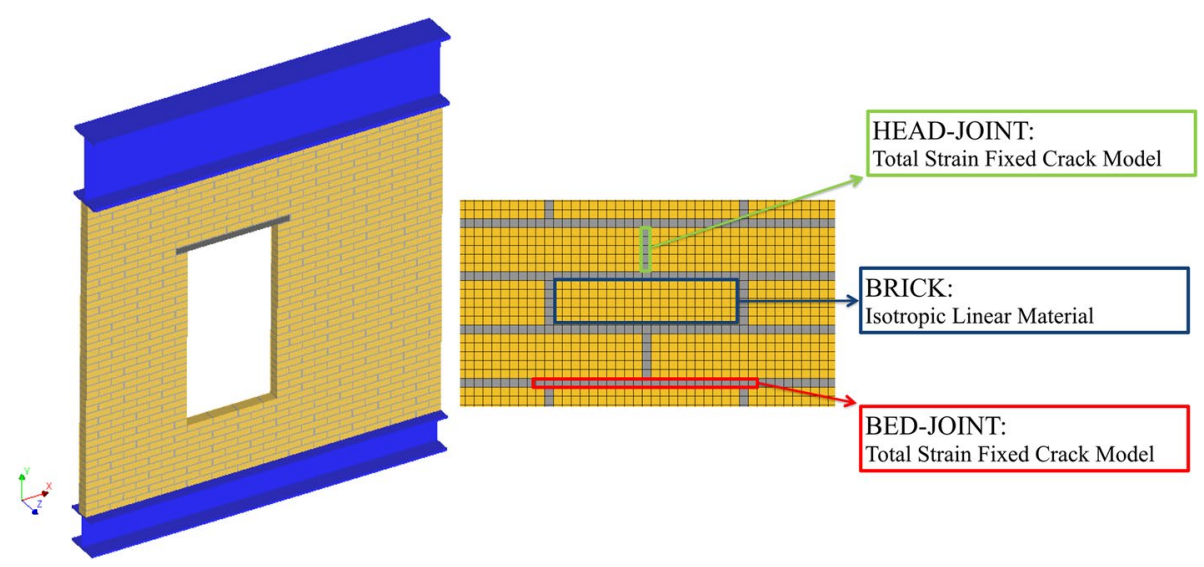

Fig. 22 3D impression of the 2D-plane stress FEM model using a micro-modelling approach 
relationship which rotates in the direction of the total strain. The reader is referred to Vecchio and Collins (1986), Rots and Blaauwendraad (1989), or Feenstra et al. (1998).

Additional properties in comparison to the macro model are:

- Dimensions: $3.07 \times 2.70 \times 0.10 \mathrm{~m}$,

- Brick size: $210 \times 50 \times 100 \mathrm{~mm}$,

- Mortar thickness: $10 \mathrm{~mm}$,

- 2D Plane Stress Model,

- 4-Node Linear Elements,

- $2 \times 2$ Integration scheme,

- Mesh size: $10 \times 10 \mathrm{~mm}$,

- Full Newton-Raphson scheme with force and displacement conditions with a $1 \%$ residual tolerance for convergence.

Since the micro model is computationally expensive, it was calibrated (in this stage) only against the monotonic backbone curve of the experiments and a stepwise loading with a single repetition.

\subsubsection{Window bank micro model (spandrel)}

Due to the reduced size of the spandrels, these were modelled only with the micro-modelling approach (see Fig. 23). As both the wall and window bank tests served for the same material calibration, these material properties are identical to those used for the full-scale wall micro models (see Table 6).

The spandrel was modelled including the contact steel plates for the supports, jacks, and counterweights. The specific model properties are listed next:

- Dimensions: $1.31 \times 0.53 \times 0.10 \mathrm{~m}$,

- 2D Plane Stress Model,

- Steel plates modelled, E=200 GPa,

- Counterweight as line force,

- Monotonic Displacement,

- Mesh size $10 \times 10 \mathrm{~mm}$.

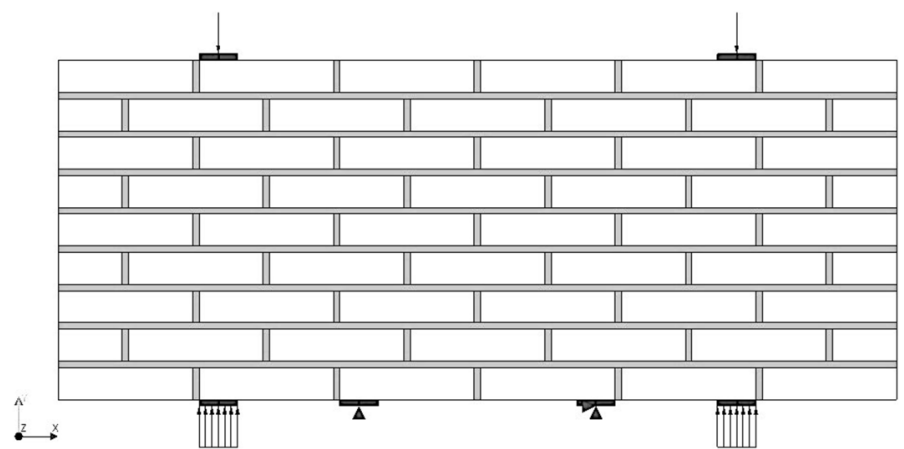

Fig. 23 Spandrel FEM model 
Table 6 Calibrated properties for the micro model

\begin{tabular}{llllll}
\hline Property & Symbol & Bed joints & Head joints & Bricks & Units \\
\hline Density & $\rho$ & $1708^{\mathrm{a}}$ & $1708^{\mathrm{a}}$ & $\mathrm{kg} / \mathrm{m}^{3}$ & \\
Young's modulus & $\mathrm{E}$ & $1000^{\mathrm{b}}$ & $4600^{\mathrm{a}}$ & $\mathrm{MPa}$ & \\
Poisson's ration & $\mathrm{a}$ & $0.14^{\mathrm{a}}$ & $0.14^{\mathrm{a}}$ & - & \\
Tensile strength & $\mathrm{f}_{\mathrm{t}}$ & $0.09^{\mathrm{a}}$ & $0.05^{\mathrm{b}}$ & $\mathrm{NA}$ & $\mathrm{MPa}$ \\
Fracture energy (tension) & $\mathrm{G}_{\mathrm{f}}^{\mathrm{I}}$ & $7.53^{\mathrm{c}}$ & $4.99^{\mathrm{c}}$ & $\mathrm{NA}$ & $\mathrm{N} / \mathrm{m}$ \\
Compressive strength & $\mathrm{f}_{\mathrm{c}}$ & $3.81^{\mathrm{a}}$ & $\mathrm{NA}$ & $\mathrm{MPa}$ & \\
Fracture energy (compression) & $\mathrm{G}_{\mathrm{C}}$ & $6400^{\mathrm{b}}$ & $\mathrm{NA}$ & $\mathrm{N} / \mathrm{m}$ & \\
Shear retention & - & Damage based $^{\mathrm{d}}$ & $\mathrm{NA}$ & - & \\
\hline
\end{tabular}

${ }^{\mathrm{a}}$ From companion tests (material characterisation)

${ }^{\mathrm{b}}$ From calibration

${ }^{\mathrm{c}}$ From formulation (Schreppers et al. 2016)

${ }^{\mathrm{d}}$ Reduction of Poisson's ratio. See Slobbe et al. (2013)

\subsection{Computational results of full-scale walls models}

The focus of the calibration efforts was on the stiffness and strength of the samples, while also capturing the observed crack pattern. In Fig. 24, the calibration against two wall specimens is summarised. It can be observed that the stiffness and strength are both well represented, that there is a loss of strength after the first unloading, and while the hysteresis of the experiments is also well depicted, the degradation observed during the repetitions (see Fig. 16) is not reproduced with the models, mainly because it is also not present in the material model. In Fig. 24a, b, d the dashed lines appear solid due to the overlay of the repetitions in the cyclic analyses. The inclusion of degradation is hence a point for future improvement.

Furthermore, monotonic analyses were performed. Here, the micro model is more stable than the macro model when applying a monotonic loading protocol (see Fig. 24c) and is capable of reaching the degraded step points better (see Fig. 24d), yet, there is barely any damaged stiffness (or plastic deformation) at the later steps (Fig. 24d); here the macro models fits the experiments more loyally (Fig. 24b), though for the case of TUD-Comp 41, the macro model overestimates the plastic deformations (Fig. 24a).

In terms of crack patterns, both models represent the three main cracks elegantly (see Fig. 25); however, for the case of TUD-Comp 41, no model shows the observed crack pattern precisely. Yet, the micro model appears closer to the experiments by displaying the staircase pattern at the bottom of the window, and while it doesn't show a horizontal crack at the top of the window, a staircase crack was observed here in some cases (see Fig. 15). This is most likely related to the issue of mesh directional bias for smeared crack band models (see e.g. Slobbe et al. 2013). The relative crack width is also closer to the experiments for the micro model in contrast to the macro model, where the narrowest crack computed was actually the largest in the test.

In sum, the micro model seems better suited for replicating the experimental test, especially when crack patterns are of importance, however, it is four times more computationally expensive and requires some additional parameters which may not be readily available. 
(a) TUD-Comp 41

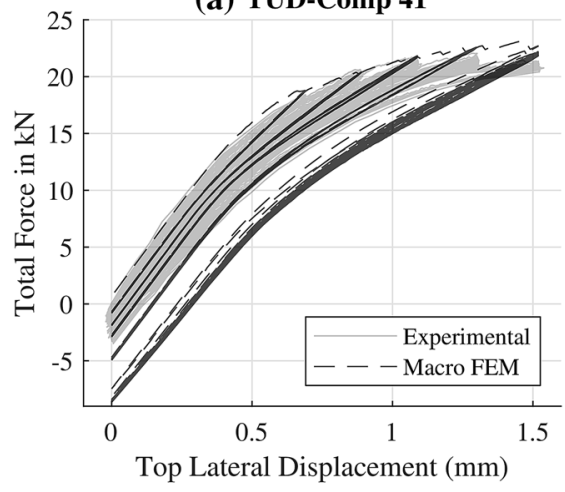

(c) Micro and Macro Models

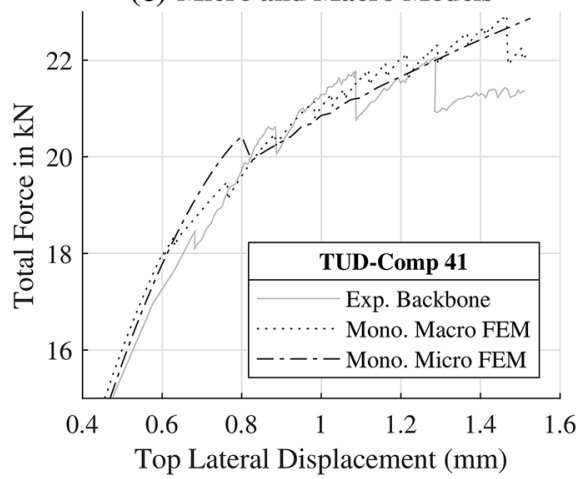

(b) TUD-Comp 42

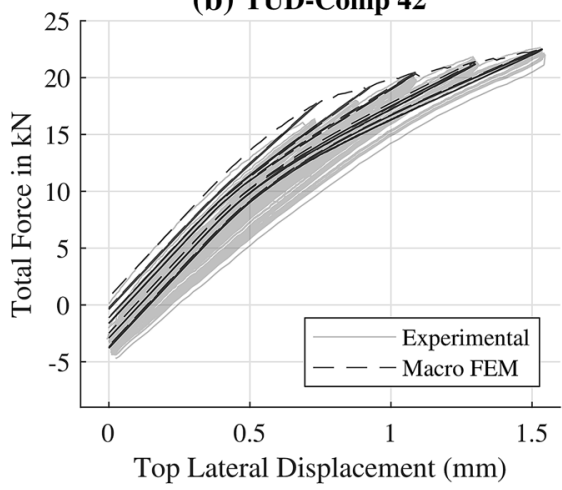

(d) TUD-Comp 41 - Micro FEM

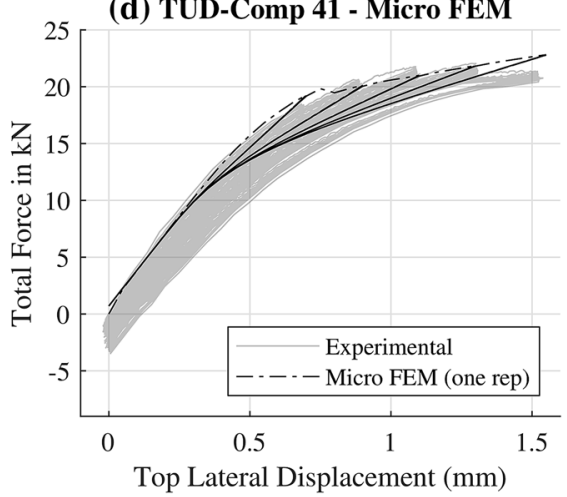

Fig. 24 Calibration force-displacement curves from the models based on two of the test walls. a, b Experimental and repetitive macro model force-displacement curves for wall TUD-Comp 41 and TUD-Comp 42, respectively; c monotonic force-displacement curve from both macro and micro models against the experimental backbone curve of wall TUD-Comp 41; and d force-displacement micro model with five steps of one repetition each against the experimental curve of wall TUD-Comp 41
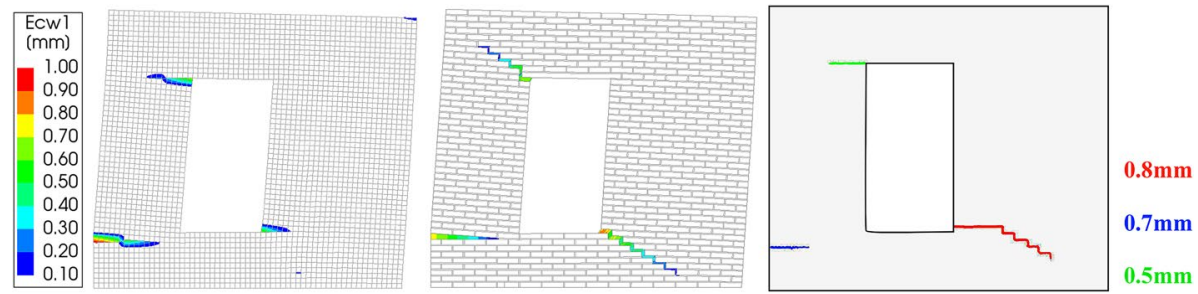

Fig. 25 Comparison between crack pattern from Macro-FEM, Micro-FEM, and TUD-Comp 41 (Exp. DIC)

\subsection{Computational results of window bank models}

The calibration of the material model was further complemented by a replication of the spandrel tests. Due to the computational expense of the micro modelling approach employed, the models were calibrated only against the monotonic spandrel tests. The 
inclusion of the repetitive tests is a point for future improvement. Moreover, if the observed degradation can be included in a homogenised material model, then a macro modelling approach may also be possible for these tests.

Nevertheless, a good agreement was achieved between the models and the experimental data (see Fig. 26). Here, the initial stiffness, the maximum strength, and the postpeak plateau were observed. In particular, the inversion point of linear to non-linear behaviour (recognisable on the logarithmic scale around $50 \mu \mathrm{m}$ of CMOD) matches the data beautifully. Note that the loss of linearity occurs at approximately half the ultimate capacity of the specimens. This was observed in the model to be related to the exceedance of crack initiation strain for the elements located at the very top of the sample. It also shows how cracks may arise long before the ultimate structural capacity is reached thus leading to the aesthetic DS1.

Further, two variants for the stiffness of the linear-elastic bricks are plotted. The lower Young's modulus corresponds to the stiffness of masonry as a composite material assigned to the brick, while the higher value of $8 \mathrm{GPa}$ belongs to the testing of individual bricks. In the models, a Young's modulus of $1 \mathrm{GPa}$ is given to the mortar elements which represent the mortar and the two mortar-brick interfaces, this value is kept the same for both variations of the brick stiffness. The latter value of $8 \mathrm{GPa}$ for the brick was observed to be slightly too stiff, hence the lower value was also used. It is possible that the poor bond of the replicated masonry leads to an even lower stiffness which is then better captured by reducing the Young's modulus of the bricks.

Additionally, the crack pattern determined by the models was also observed in some of the monotonic tests as is shown in Fig. 27. The spread of the local material property values will affect the precise crack pattern in both experiments and computations.
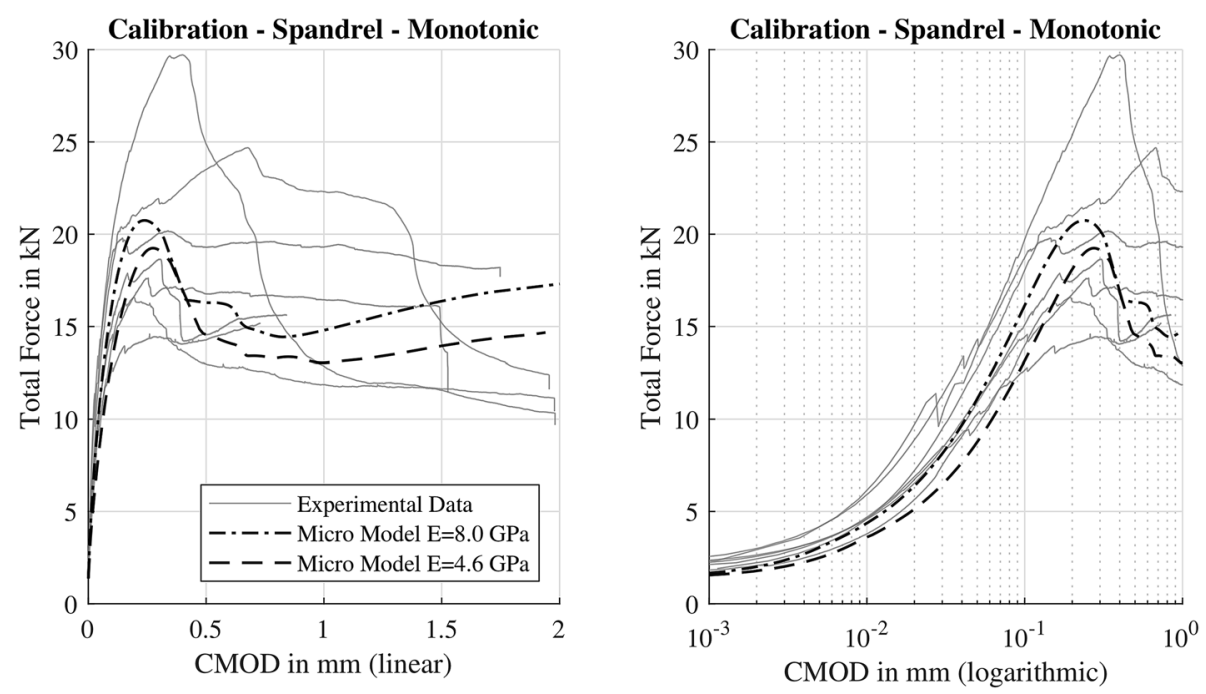

Fig. 26 Calibration of a Window Bank finite element model against the monotonic spandrel tests plotted on linear (left) and logarithmic (right) scales 
Fig. 27 Comparison of crack pattern against one of the monotonic spandrel tests

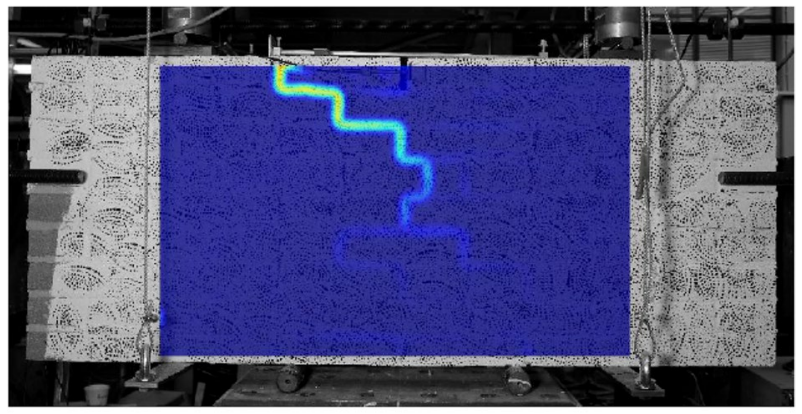

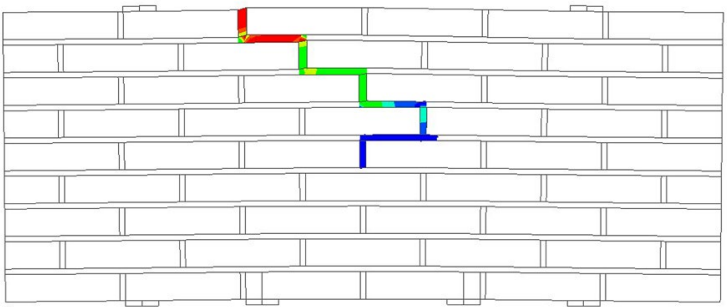

\section{Conclusions}

It is first important to emphasise that the conclusions provided here are derived from the representative, albeit limited cases treated in this study. They are applicable insofar the geometry, excitations, and material do not deviate significantly from the studied cases. The results are presented for the case of baked-clay, single-wythe masonry walls without important interaction with the rest of a structure and subjected to in-plane loads. A limited number of walls has been tested experimentally and models have been calibrated against these tests.

With the goal of observing damage in these masonry walls, a new, light damage characterisation was defined based on the number and width of cracks ( $\Psi$ as damage parameter). Damage was thus summarised into a single number, which allowed for the observation and quantification of the initiation and propagation of damage between samples and computational models.

Upon this damage definition, light damage (DS1) was experimentally observed in the range of lateral drift values of $0.3 \%$ and $1.1 \%$. Masonry walls subjected to drift values below $0.3 \%$ o are not expected to display any form of noticeable damage; walls that have drifted above $1.1 \%$ o are expected to exhibit cracks wider than $4 \mathrm{~mm}$ and will have exceeded DS1.

Paired with the geometry-dependent drift limits proposed by Messali and Rots (2018) for the ultimate limit state, the serviceability drift limits presented in this paper can be expanded to different wall geometries to obtain a more complete picture of the seismic performance of masonry for the light damage states.

During laboratory tests a degradation of the material was observed: the repetition of a certain prescribed displacement was followed by a reduction in the required force ranging from $4 \%$ to $22 \%$. This is linked to the propagation of cracks. Cracks propagating horizontally through the bed joint lead to a lower reduction compared to cracks propagating vertically in masonry, zigzagging through bed and head joints.

A material model considering the non-linear orthotropy and directionality of masonry (the Engineering Masonry Model) was well suited when reproducing the stiffness, strength and crack configuration of the tested walls. However, the degradation observed in the experiments was not reproduced well. Extending the finite element material model to include this degradation is a logical next step. 
Further, Digital Image Correlation (DIC) was successfully employed to track the propagation of cracks throughout the tested masonry walls. Here, improvements to the pattern and its application, and to the analysis of the images, allowed for an accurate representation of the cracks' widths and lengths.

Finally, to expand the observations yielded in this study, additional specimen geometry, configuration, and materials need to be explored. Specimen pre-damage is also to be investigated experimentally to determine whether existing cracks modify the observed crack patterns. Together with DIC and calibrated finite element models including the observed degradation, the behaviour of cracks and light damage states in full-scale masonry can be further characterised.

Acknowledgements This research was funded by Nederlandse Aardolie Maatschappij (NAM) under contract number UI67339 'Damage sensitivity of Groningen masonry building structures-Experimental and computational studies', contract holders: Jan van Elk and Jeroen Uilenreef. This cooperation is gratefully acknowledged. The authors also express their gratitude to Piet van Staalduinen for participating in this project and linking it to damage surveys, and to Beatriz Zapico Blanco from Arup for reviewing tasks.

Open Access This article is distributed under the terms of the Creative Commons Attribution-NonCommercial 4.0 International License (http://creativecommons.org/licenses/by-nc/4.0/), which permits any noncommercial use, duplication, adaptation, distribution and reproduction in any medium or format, as long as you give appropriate credit to the original author(s) and the source, a link is provided to the Creative Commons license and any changes made are indicated.

\section{Appendixes}

\section{Appendix 1: Expressions for the light damage parameter $\Psi$}

\begin{tabular}{|c|c|c|c|}
\hline Equation & \multicolumn{2}{|l|}{ Description } & Expression \\
\hline 1 & \multicolumn{2}{|c|}{ Expression for $\Psi$ for one wall } & $\Psi=2 \cdot \mathrm{n}_{\mathrm{c}}^{0.15} \cdot \hat{\mathrm{c}}_{\mathrm{w}}^{0.3}$ \\
\hline 2 & \multicolumn{2}{|c|}{ Weighted crack width for one wall (part of Eq. 1) } & $\hat{\mathrm{c}}_{\mathrm{w}}=\frac{\sum_{\mathrm{i}-1}^{\mathrm{n}_{\mathrm{c}}} \mathrm{c}_{\mathrm{w}, \mathrm{i}}^{2} \cdot \mathrm{c}_{\mathrm{L}, \mathrm{i}}}{\sum_{\mathrm{i}-1}^{\mathrm{n}_{1}} \mathrm{c}_{\mathrm{w}, \mathrm{i}} \cdot \mathrm{c}_{\mathrm{L}, \mathrm{i}}}$ \\
\hline 3 & \multicolumn{2}{|c|}{ Psi for a full structure consisting of $\mathrm{N}$ walls } & $\bar{\Psi}=\frac{\sum_{\mathrm{i}=1}^{\mathrm{N}} \Psi_{\mathrm{i}} \cdot \mathrm{A}_{\mathrm{i}}}{\mathrm{A}_{\mathrm{T}}}$ \\
\hline 4 & \multicolumn{2}{|c|}{ Absolute damage for comparison Psi between walls } & $\Psi_{\mathrm{i}}^{\prime}=\Psi_{\mathrm{i}} \cdot \frac{\mathrm{A}_{\mathrm{i}}}{\mathrm{A}_{\mathrm{m}}}$ \\
\hline 5 & \multicolumn{2}{|c|}{ Cost of repair of a structure } & $\mathrm{Z}=\mathrm{Z}_{0}+\bar{\Psi} \cdot \mathrm{A}_{\mathrm{T}} \cdot \zeta$ \\
\hline Symbol & Units & \multicolumn{2}{|c|}{ Description } \\
\hline$\Psi$ & - & \multicolumn{2}{|c|}{ Psi damage parameter } \\
\hline $\mathrm{n}_{\mathrm{c}}$ & - & \multicolumn{2}{|c|}{ Number of cracks on a wall } \\
\hline$\hat{\mathrm{c}}_{\mathrm{w}}$ & $\mathrm{mm}$ & \multicolumn{2}{|c|}{ Weighted crack width } \\
\hline $\mathrm{c}_{\mathrm{w}}$ & $\mathrm{mm}$ & \multicolumn{2}{|c|}{ Crack width (of crack i) } \\
\hline$c_{L}$ & $\mathrm{~mm}$ & \multicolumn{2}{|c|}{ Crack length (of crack i) } \\
\hline $\bar{\Psi}$ & - & \multicolumn{2}{|c|}{ Psi of multiple walls } \\
\hline $\mathrm{N}$ & - & \multicolumn{2}{|c|}{ Number of walls } \\
\hline$A_{i}$ & {$[\mathrm{~L}]^{2}$} & \multicolumn{2}{|c|}{ Area of wall i } \\
\hline$\Psi_{\mathrm{i}}$ & - & \multicolumn{2}{|c|}{ Psi of wall i } \\
\hline$A_{T}$ & {$[\mathrm{~L}]^{2}$} & \multicolumn{2}{|c|}{ Total area of walls considered } \\
\hline
\end{tabular}




\begin{tabular}{lll}
\hline Symbol & Units & Description \\
\hline$\Psi_{\mathrm{i}}^{\prime}$ & - & Comparison Psi for wall i \\
$\mathrm{A}_{\mathrm{m}}$ & - & Mean area of walls considered \\
$\mathrm{Z}$ & {$[€]$} & Total cost of repair \\
$\mathrm{Z}_{0}$ & {$[€]$} & Base or mobilisation cost for repairs \\
$\zeta$ & {$[€] /[\mathrm{L}]^{2}$} & Unitary cost for repair per unit of Psi \\
\hline
\end{tabular}

\section{Appendix 2}

See Table 7.

Table 7 Overview of masonry properties as used in this study

\begin{tabular}{|c|c|c|c|c|c|c|c|c|}
\hline & & & \multicolumn{3}{|c|}{$\begin{array}{l}\text { Campaign of } 2016 \\
\text { Poor clay-brick masonry }\end{array}$} & \multicolumn{3}{|c|}{$\begin{array}{l}\text { Campaign of } 2017 \\
\text { Very poor clay-brick M. }\end{array}$} \\
\hline & & & Average & SD & C.o.V. & Average & SD & C.o.V. \\
\hline Compressive strength of mortar & $\mathrm{f}_{\mathrm{m}}$ & $\mathrm{MPa}$ & 3.81 & 0.34 & 0.09 & 3.84 & 0.43 & 0.11 \\
\hline Flexural strength of mortar & $\mathrm{f}_{\mathrm{mt}}$ & $\mathrm{MPa}$ & 1.40 & 0.17 & 0.12 & 1.57 & 0.11 & 0.07 \\
\hline $\begin{array}{l}\text { Normalized compressive strength of } \\
\text { element prescribed by producer }\end{array}$ & $\mathrm{f}_{\mathrm{b}}$ & $\mathrm{MPa}$ & 28.31 & 2.92 & 0.10 & & & \\
\hline Flexural strength of brick & $\mathrm{f}_{\mathrm{bt}}$ & $\mathrm{MPa}$ & 6.31 & 0.72 & 0.11 & & & \\
\hline $\begin{array}{l}\text { Elastic modulus of brick from bend- } \\
\text { ing test }\end{array}$ & $\mathrm{E}_{\mathrm{b}}$ & $\mathrm{MPa}$ & 8049 & 423 & 0.05 & & & \\
\hline $\begin{array}{l}\text { Compressive strength of masonry in } \\
\text { the direction perpendicular to bed } \\
\text { joints }\end{array}$ & $\mathrm{f}_{\mathrm{m}}^{\prime}$ & $\mathrm{MPa}$ & 14.02 & 0.56 & 0.04 & 11.35 & 0.83 & 0.07 \\
\hline \multirow{3}{*}{$\begin{array}{l}\text { Elastic modulus of masonry in the } \\
\text { direction perpendicular to bed joints }\end{array}$} & $\mathrm{E}_{1}$ & $\mathrm{MPa}$ & 4380 & 605 & 0.14 & 2919 & 442 & 0.15 \\
\hline & $\mathrm{E}_{2}$ & $\mathrm{MPa}$ & 4068 & 783 & 0.19 & 2731 & 732 & 0.27 \\
\hline & $\mathrm{E}_{3}$ & $\mathrm{MPa}$ & 4590 & 603 & 0.13 & 3087 & 315 & 0.10 \\
\hline $\begin{array}{l}\text { Poisson ratio of masonry in the direc- } \\
\text { tion perpendicular to bed joints }\end{array}$ & $v$ & - & 0.14 & 0.02 & 0.11 & 0.14 & 0.004 & 0.03 \\
\hline $\begin{array}{l}\text { Fracture energy in compression for } \\
\text { loading perpendicular to bed joints }\end{array}$ & $\mathrm{G}_{\mathrm{f}-\mathrm{c}}$ & $\mathrm{N} / \mathrm{mm}$ & 28.52 & 3.4 & 0.12 & 26.05 & 3.15 & 0.12 \\
\hline $\begin{array}{l}\text { Compressive strength of masonry in } \\
\text { the direction parallel to bed joints }\end{array}$ & $\mathrm{f}_{\mathrm{m}, \mathrm{h}}$ & $\mathrm{MPa}$ & 13.11 & 2.41 & 0.18 & & & \\
\hline \multirow{3}{*}{$\begin{array}{l}\text { Elastic modulus of masonry in the } \\
\text { direction parallel to bed joints }\end{array}$} & $\mathrm{E}_{1, \mathrm{~h}}$ & $\mathrm{MPa}$ & 3332 & 565.00 & 0.17 & & & \\
\hline & $\mathrm{E}_{2, \mathrm{~h}}$ & $\mathrm{MPa}$ & 3664 & 689.00 & 0.19 & & & \\
\hline & $\mathrm{E}_{3, \mathrm{~h}}$ & $\mathrm{MPa}$ & 3207 & 592.00 & 0.18 & & & \\
\hline $\begin{array}{l}\text { Fracture energy in compression for } \\
\text { loading parallel to bed joints }\end{array}$ & $\mathrm{G}_{\mathrm{f}-\mathrm{c}, \mathrm{h}}$ & $\mathrm{N} / \mathrm{mm}$ & 35.1 & 6.63 & 0.19 & & & \\
\hline $\begin{array}{l}\text { Masonry bending strength with the } \\
\text { moment vector parallel to the bed } \\
\text { joints and in the plane of the wall }\end{array}$ & $f_{x 1}$ & $\mathrm{MPa}$ & 0.16 & 0.03 & 0.18 & & & \\
\hline Youngs modulus from OOP1 & $\mathrm{E}_{\mathrm{fx} 1}$ & $\mathrm{MPa}$ & 3756 & 1789 & 0.21 & & & \\
\hline $\begin{array}{l}\text { Masonry bending strength with the } \\
\text { moment vector orthogonal to the } \\
\text { bed joint and in the plane of the } \\
\text { wall }\end{array}$ & $\mathrm{f}_{\mathrm{x} 2}$ & $\mathrm{MPa}$ & 0.65 & 0.17 & 0.25 & & & \\
\hline
\end{tabular}


Table 7 (continued)

\begin{tabular}{|c|c|c|c|c|c|c|c|c|}
\hline & & & \multicolumn{3}{|c|}{$\begin{array}{l}\text { Campaign of } 2016 \\
\text { Poor clay-brick masonry }\end{array}$} & \multicolumn{3}{|c|}{$\begin{array}{l}\text { Campaign of } 2017 \\
\text { Very poor clay-brick M. }\end{array}$} \\
\hline & & & Average & SD & C.o.V. & Average & SD & C.o.V. \\
\hline Youngs modulus from OOP2 & Efx2 & $\mathrm{MPa}$ & 7080 & 593 & 0.08 & & & \\
\hline $\begin{array}{l}\text { Masonry bending strength with the } \\
\text { moment vector orthogonal to the } \\
\text { plane of the wall }\end{array}$ & $f_{x 3}$ & $\mathrm{MPa}$ & 0.46 & 0.09 & 0.20 & 0.35 & 0.14 & 0.40 \\
\hline Youngs modulus from IP bending & $\mathrm{E}_{\mathrm{fx} 3}$ & $\mathrm{MPa}$ & 2924 & 480 & 0.16 & 2084 & 526 & 0.25 \\
\hline Flexural bond strength & $\mathrm{f}_{\mathrm{w}}$ & $\mathrm{MPa}$ & 0.15 & 0.05 & 0.32 & 0.09 & 0.03 & 0.35 \\
\hline $\begin{array}{l}\text { Masonry (bed joint) initial shear } \\
\text { strength }\end{array}$ & $\mathrm{f}_{\mathrm{v} 0}$ & $\mathrm{MPa}$ & 0.20 & & & 0.14 & & \\
\hline $\begin{array}{l}\text { Masonry (bed joint) shear friction } \\
\text { coefficient }\end{array}$ & $\mu$ & & 0.69 & & & 0.79 & & \\
\hline $\begin{array}{l}\text { Residual masonry (bed joint) initial } \\
\text { shear strength }\end{array}$ & $\mathrm{f}_{\mathrm{v} 0 \text {, res }}$ & $\mathrm{MPa}$ & 0.05 & & & 0.03 & & \\
\hline $\begin{array}{l}\text { Residual masonry (bed joint) shear } \\
\text { friction coefficient }\end{array}$ & $\mu_{\rho \varepsilon \sigma}$ & & 0.60 & & & 0.71 & & \\
\hline
\end{tabular}

\section{References}

Abo-El-Ezz A, Nollet M, Nastev M (2013) Seismic fragility assessment of low-rise stone masonry buildings. Earthq Eng Eng Vib 12:87-97

Arup (2013) Groningen 2013-implementation study. Arup, London

Bal IE, Dais D, Smyrou E (2018) "Differences" between induced and natural seismic events. In: 16th European conference on earthquake engineering, Thessaloniki 18-21 June, 2018

Beyer K, Mergos P (2015) Sensitivity of drift capacities of URM walls to cumulative damage demands and implications on loading protocols for quasi-static cyclic tests. In: 12th North American Masonry Conference, Denver, Colorado May 17-20, 2015

Bommer JJ, Stafford PJ, Ntinalexis M (2017) Empirical ground-motion prediction equations for peak ground velocity from small-magnitude earthquakes in the Groningen field using multiple definitions of the horizontal component of motion. NAM, Assen

Boscardin MD, Cording EJ (1989) Building response to excavation-induced settlement. J Geotech Eng 115(1):1-21

Burland JB, Wroth CP (1974) Settlement of buildings and associated damage. In: Proceedings of conference on settlement of structures, Cambridge. Pentech Press, pp 611-654

Castellazzi G, D’Altri AM, de Miranda S, Chiozzi A, Tralli A (2017) Numerical insights on the seismic behaviour of a non-isolated historical masonry tower. Bull Earthq Eng. https://doi.org/10.1007/ s10518-017-0231-6

Colangelo F (2015) On the code-compliant verification of seismic damage to non-structural masonry infills. Bull Earthq Eng 13:2051-2072. https://doi.org/10.1007/s10518-014-9704-z

Crammond G, Boyd SW, Dulieu-Barton JM (2013) Speckle pattern quality assessment for digital image correlation. Opt Lasers Eng 51(2013):1368-1378

Crowley H, Pinho R, van Elk J, Uilenreef J (2018) Probabilistic damage assessment of buildings due to induced seismicity. Bull Earthq Eng. https://doi.org/10.1007/s10518-018-0462-1

de Vent I, Rots JG, van Hees RPJ (2011) Structural damage in masonry-developing diagnostic decision support. TU Delft, Delft

Didier M, Abbiati G, Broccardo M, Beyer K, Danciu L, Petrović M, Mojsilović N, Stojadinović B (2017) Quantification of non-structural damage in unreinforced masonry walls induced by geothermal reservoir exploration using quasi-static cyclic tests. In: Proceedings of the 13th Canadian Masonry Symposium, Halifax, Canada, June 2017

Didier M, Abbiati G, Hefti F, Broccardo M, Stojadinovic B (2018) Damage quantification in plastered unreinforced masonry walls using digital image correlation. In: 10th Australasian Masonry Conference, 14-18 February, 2018 
Dyer BC, Schanz U, Spillmann T, Ladner F, Häring MO (2008) Microseismic imaging of a geothermal reservoir stimulation. Lead Edge 27(7):856-869

Elmenshawi AE, Sorour M, Mufti A, Jaeger LG, Shrive N (2010) In-plane seismic behaviour of historic stone masonry. Can J Civ Eng 37(3):465-476

Esposito R, Terwel K, Ravenshorst GJP, Schipper HR, Messali F, Rots JG (2016) Cyclic pushover test on an unreinforced masonry structure resembling a typical Dutch terraced house. TU Delft, Delft

Esposito R, Messali F, Ravenshorst GJP, Schipper HR, Rots JG (2018) Seismic assessment of a twostorey unreinforced masonry Dutch terraced house. Bull Earthq Eng (under review)

Feenstra PH, Rots JG, Arnesen A, Teigen JG, Hoiseth KV (1998) A 3D constitutive model for concrete based on a co-rotational concept. In: Proceedings of Euro-C conference computational modelling of concrete structures, Balkema

Gattesco N, Macorini L, Dudine A (2016) Experimental response of brick-masonry spandrels under inplane cyclic loading. ASCE J Struct Eng 142(2):04015146

Giardina G, van de Graaf AV, Hendriks MAN, Rots JG, Marini A (2013a) Numerical analysis of a masonry façade subject to tunnelling-induced settlements. Eng Struct 54(2013):234-247

Giardina G, Rots JG, Hendriks MAN (2013b) Modelling of settlement induced building damage. TU Delft, Delft

Graziotti F, Tomassetti U, Kallioras S, Penna A, Magenes G (2017) Shaking table test on a full scale URM cavity wall building. Bull Earthq Eng 15(12):5329-5364

Grünthal G (1998) European Macroseismic Scale 1998 (EMS-98). European Seismological Commission, sub commission on Engineering Seismology, Working Group Macroseismic Scales. Conseil de l'Europe, Cahiers du Centre Européen de Géodynamique et de Séismologie, vol 15, Luxembourg

Jafari S, Esposito R (2019) Summary of material properties of existing Dutch masonry-updated URM abacus (version 2). Delft University of Technology. Report number C31B67WP1-16, version 02

Jafari S, Esposito R, Rots JG, Messali F (2017) Characterizing the material properties of Dutch unreinforced masonry. Procedia Eng 193:250-257

Korswagen PA, Jonkman SN, Terwel K (2019) Probabilistic assessment of structural damage from coupled multi-hazards. Struct Saf 76:135-148. https://doi.org/10.1016/j.strusafe.2018.08.001

Kruiver PP, van Dedem E, Romijn R, de Lange G, Korff M, Stafleu J, Gunnink JL, Rodriguez-Marek A, Bommer JJ, van Elk J, Doornhof D (2017) An integrated shear-wave velocity model for the Groningen gas field, The Netherlands. Bull Earthq Eng 15:3555-3580. https://doi.org/10.1007/s1051 8-017-0105-y

Laurin F, Charrier JS, Lévêque D, Maire JF, Mavel A, Nuñez P (2012) Determination of the properties of composite materials thanks to digital image correlation measurements. Procedia IUTAM 4(2012):106-115

Lourenço PB (1996) Computational strategies for masonry structures. PhD Dissertation, TU Delft

Lourenço PB, Rots JG (1997) Multisurface interface model for analysis of masonry structures. J Eng Mech 123(7):660-668

Mergos P, Beyer K (2014) Loading protocols for European regions of low to moderate seismicity. Bull Earthq Eng 12:2507-2530. https://doi.org/10.1007/s10518-014-9603-3

Mergos P, Beyer K (2015) Loading protocols for structures designed for different behaviour factors. In: SECED 2015 conference: earthquake risk and engineering towards a resilient world 9-10 July 2015, Cambridge, UK

Messali F, Rots JG (2018) In-plane drift capacity at near collapse of rocking unreinforced calcium silicate and clay masonry piers. Eng Struct 164(2018):183-194

Messali F, Ravenshorst GJP, Esposito R, Rots JG (2017) Large-scale testing program for the seismic characterization of Dutch masonry walls. In: 16th World Conference on earthquake, WCEE 2017, 9-13 Jan, Santiago, Chile

Mignan A, Landtwing D, Kästli P, Mena B, Wiemer S (2015) Induced seismicity risk analysis of the 2006 Basel, Switzerland, Enhanced Geothermal System project: influence of uncertainties on risk mitigation. Geothermics 53(2015):133-146

Mojsilović N, Salmanpour AH (2016) Masonry walls subjected to in-plane cyclic loading: application of Digital Image Correlation for deformation field measurement. Institute of Structural Engineering, Department of Civil, Environmental and Geomatic Engineering, ETH Zurich, 8093, Switzerland

NAM (2013) Technical Addendum to the Winningsplan Groningen 2013 - subsidence, induced earthquakes and seismic hazard analysis in the Groningen field. NAM, Assen

NAM (2016) Production, subsidence, induced earthquakes and seismic hazard and risk assessment in the Groningen Field. NAM, Technical Addendum to the Winningsplan Groningen 2016. www.NAM.nl

Noorlandt R, Kruiver PP, de Kleine MPE, Karaoulis M, de Lange G, di Matteo A, von Ketlehodt J, Ruigrok E, Edwards B, Rodriguez-Marek A, Bommer JJ, van Elk J, Doornhof D (2018) Characterisation 
of ground motion recording stations in the Groningen gas field. J Seismol 2018(22):605-623. https ://doi.org/10.1007/s10950-017-9725-6

Österberg G (1935) Topography of the layer of rods and cones in the human retina. Acta Ophthalmol [Suppl] 13(6): 1-102

Ramos T, Furtado A, Eslami S, Alves S, Rodrigues H, Arêde A, Tavares P, Moreira P (2015) 2D and 3D Digital Image correlation in civil engineering-measurements in a masonry wall. Procedia Eng 114(2015):215-222

Rots JG, Blaauwendraad J (1989) Crack models for concrete-discrete, smeared, fixed, multi-directional or rotating?. Heron 34. ISSN: 0046-7316

Rots JG, vd Pluijm R, Vermeltfoort AT (1994) CUR Report 171, CUR, Gouda (Dutch version). See Rots et al., (1997) for english translation

Rots JG, vd Pluijm R, Vermeltfoort AT (1997) Structural masonry—an experimental/numerical basis for practical design rules. Balkema. ISBN: 9054106808

Rots JG, Messali F, Esposito R, Jafari S, Mariani V (2016) Computational modelling of masonry with a view to groningen induced seismicity. In: 10th SAHC structural analysis of historical construction, Leuven

Schreppers GMA, Garofano A, Messali F, Rots JG (2016) DIANA validation report for masonry modelling. DIANA FEA Report 2016-DIANA-R1601 TU Delft Structural Mechanics Report CM-2016-17

Slobbe AT, Hendriks MAN, Rots JG (2013) Systematic assessment of directional mesh bias with periodic boundary conditions: applied to the crack band model. Eng Fract Mech 109:186-208

Spetzler J, Dost B (2017) probabilistic seismic hazard analysis for induced earthquakes in Groningenupdate. KNMI Report, PSHA Groningen, update

Theil H (1950) A rank-invariant method of linear and polynomial regression analysis. I, II, III. Nederl. Akad. Wetensch., Proc., 53: 386-392, 521-525, 1397-1412, MR 0036489

Tomassetti U, Kallioras S, Graziotti F, Correia AA (2017) Preliminary report on the construction of the building prototype at the LNEC laboratory. Lisbon, March 2017

Van Staalduinen P, Terwel K, Rots JG (2018) Onderzoek naar de oorzaken van bouwkundige schade in Groningen Methodologie en case studies ter duiding van de oorzaken. Delft University of Technology. Report number CM-2018-01, 11 July 2018. www.NationaalCoordinatorGroningen.nl

Vecchio FJ, Collins MP (1986) The modified compression field theory for reinforced concrete elements subjected to shear. ACI J 83(2):219-231

Publisher's Note Springer Nature remains neutral with regard to jurisdictional claims in published maps and institutional affiliations. 